\title{
RDUS
}

Revue de DROIT UNIVERSITÉ DE SHERBROOKE

Titre :

Auteur(s) :

Revue :

Pages :

ISSN :

Éditeur :

URI :

DOI :

\section{L'EXPERTISE ET LA CONTRE-EXPERTISE EN MATIÈRE FAMILIALE}

Christine BENOÎT, Karina PIGEON

RDUS, 1994-1995, volume 25, numéro 1-2

$175-235$

$0317-9656$

Université de Sherbrooke. Faculté de droit.

http://hdl.handle.net/11143/12913

https://doi.org/10.17118/11143/12913 
Page vide laissée intentionnellement. 
$\underline{\text { ARTICLE }}$

\title{
L'EXPERTISE ET LA CONTRE-EXPERTISE EN MATIĖRE FAMILIALE*
}

\author{
par Christine BENOÎT** \\ et Karina PIGEON***
}

L'aide considérable apportée par la preuve par expert dans les litiges en matière familiale rend l'importance de ce moyen de preuve incontestable. Le texte qui suit jette un regard critique sur cette preuve particulière. Il y sera question, entre autres, du rôle de l'expert dans les litiges familiaux, des limites légales et scientifiques de l'expertise ainsi que de la recevabilité et de l'admissibilité de la preuve par expert. En terminant, nous proposons des solutions aux problèmes les plus fréquemment rencontrés, notamment, concernant la problématique de la multiplication des expertises ainsi que le problème des expertises contradictoires.

The importance of expert testimony in matters of family law is obvious. This article examines the role of expert witnesses in domestic litigation, its legal and scientific limits and, in general, the admissibility of expert testimony. The writers also propose solutions to problems most often encountered in this regard, including those resulting from the growing trend to having recourse to experts as well as the problems arising out of contradictory expert opinions.

1. Le présent texte s'est mérité le premier prix du concours juridique «Charles-Coderre 1994». La Fondation Charles-Coderre vise à promouvoir la recherche en droit ayant un impact social. Les auteurs ont révisé leur exposé en date de juillet 1995 afin d'y apporter, le cas échéant, les corrections appropriées. La Fondation Charles-Coderre a autorisé la publication de cette recherche dans la Revue de Droit Université de Sherbrooke.

2. Avocate, contentieux des Centres jeunesse de Montréal.

3. Avocate. 


\section{SOMMAIRE}

INTRODUCTION

\section{PREMIÈRE PARTIE : LE CADRE GÉNÉRAL DE}

L'EXPERTISE .............. 183

CHAPITRE I : LE DROIT À L'EXPERTISE ................. 184

Section 1: L'intérêt requis pour demander une expertise et

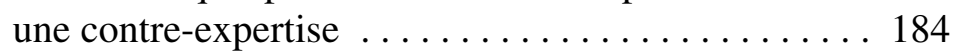

Section 2: La personne contraignable à l'expertise . . . . . . 187

A) Le principe : l'absence d'obligation de se soumettre à l'expertise . . . . . . . . . . . . . . . . . . . 187

B) Les exceptions à l'«incontraignabilité» de l'expertise . . . . . 189

1. Les exceptions au principe de l'inviolabilité de la personne humaine ........................ 189

a) L'expertise imposée par la loi . . . . . . . . . 190

b) Le pouvoir du tribunal et du directeur de la protection de la jeunesse en matière d'expertise . . . . 191

2. Le droit à la contre-expertise ............... 193

CHAPITRE II : CERTAINES CATÉGORIES D'EXPERTISE . . . . 193

Section 1 : L'expertise portant sur des faits révélés en cour . . . . . 193

Section 2 : L'expertise fondée sur les données recueillies hors cour ........................ 195

A. Les tests d'évaluation psychologique $\ldots \ldots \ldots \ldots \ldots \ldots 195$

1. L'évaluation des capacités intellectuelles ........... 195

2. L'évaluation de la compétence parentale ........... 197

3. L'évaluation des besoins psychologiques et affectifs de l'enfant .......................... 198

B) L'évaluation de la capacité de l'enfant à rendre témoignage ............................... 199

CHAPITRE III : LES LIMITES DE L'EXPERTISE _.......... 199

Section 1: $\quad$ Les limites inhérentes au droit . . . . . . . . 200

A) L'expertise restreinte aux sujets relevant de la compétence de l'expert ............................. 200 
B) L'expertise restreinte par le secret professionnel . . . . . 201

1. Les informations obtenues par l'expert protégées par

le secret professionnel . . . . . . . . . . . . . 201

a) Les critères d'application du secret professionnel . . 201

b) La renonciation volontaire au secret professionnel . . 202

2. Les informations non soumises au secret professionnel .. 203

Section 2: Les limites inhérentes à la science . . . . . . . . . 204

A) Les limites intrinsèques à la validité des tests . . . . . . . . . 204

B) Les limites concernant les variables incontrôlables . . . . 206

1. Les conceptions du professionnel . . . . . . . . . 206

2. L'attitude de la personne soumise à l'expertise . . . . . . 207

Section 3: $\quad$ La conciliation du droit et de la science ........ 208

DEUXIÈME PARTIE : L'INFLUENCE DE L'EXPERTISE

SUR L'ADJUDICATION D'UN

LITIGE EN MATIÈRE FAMILIALE . 209

\section{CHAPITRE I : LA RECEVABILITÉ DE LA PREUVE PAR}

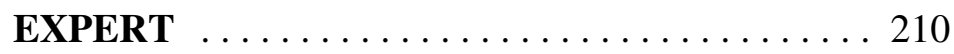

Section 1: La notion de recevabilité de la preuve par expert . . . 211

Section 2 : Les critères de recevabilité de l'expertise . . . . . . . 212

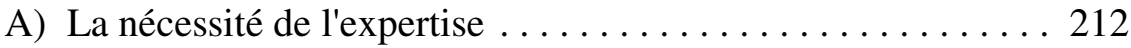

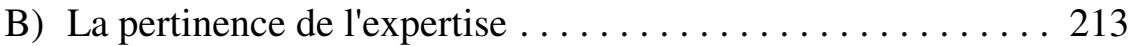

Section 3: Les conditions de forme de l'expertise ......... 214

A) Les règles générales en matière familiale . . . . . . . . 214

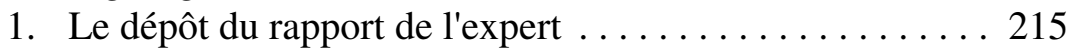

2. La preuve de l'opportunité .............. 216

3. L'accord des parties . .................. 216

B) Les règles particulières à la Loi sur la protection de

la jeunesse ...................... 216

1. Le principe : la situation de compromission ....... 217

2. L'exception : le consentement des parties ........ 218

\section{CHAPITRE II : L'ADMISSIBILITÉ DE LA PREUVE PAR}

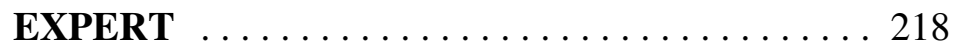

Section 1: $\quad$ La force probante de l'expertise ........... 219

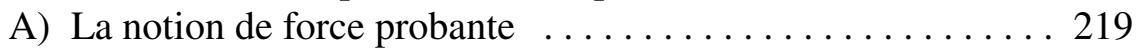


B) Les facteurs à considérer pour déterminer la force

probante . . . . . . . . . . . . . . . . . . . . . . 219

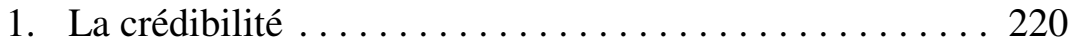

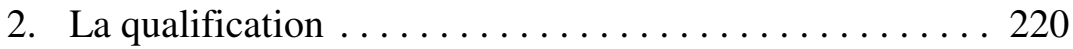

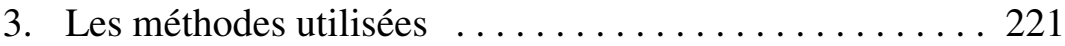

4. Les qualités de l'expertise ............... 222

5. L'opinion du témoin expert . ............. 223

6. Le témoignage sur les déclarations extrajudiciaires de l'enfant ....................... 223

a) Devant le tribunal de droit commun . . . . . . . . . . . 224

b) Devant la Chambre de la jeunesse . . . . . . . . . 224

7. La confection du rapport : distinction entre les faits

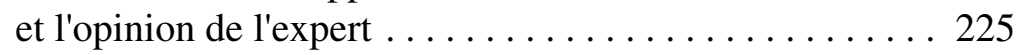

Section 2: La contradiction entre experts . . . . . . . . 225

A) Les explications possibles des expertises contradictoires . . . 226

B) Les moyens à la disposition du juge en cas d'expertises contradictoires ................ 226

Section 3: $\quad$ Le degré de preuve requis pour donner effet à l'expertise . . . . . . . . . . . . . . 227

Section 4: L'utilisation de l'expertise par les tribunaux ...... 228

TROISIÈME PARTIE : POUR UNE MEILLEURE UTILISATION DE L'EXPERTISE : CRITIQUES CONSTRUCTIVES EN REGARD DU DROIT ÉTRANGER . . 229

\section{CHAPITRE I : L'IMPACT NÉGATIF D'UN TROP GRAND} NOMBRE D'EXPERTISES $\ldots \ldots \ldots \ldots \ldots 230$

Section 1: Précisions sur la problématique de la multiplication des expertises . . . . . . . . . 230

Section 2: Le pouvoir du juge de limiter le nombre d'expertises ..................... 231

Section 3 : Le rapport de l'expert tenant lieu de preuve en lieu et place de son témoignage $\ldots \ldots \ldots \ldots \ldots \ldots 231$

\section{CHAPITRE II : L'INCERTITUDE RÉSULTANT DE}


L'ABSENCE DE DIRECTIVES IDENTIQUES PRÉALABLES DONNÉES AUX EXPERTS . . . 232

CHAPITRE III : LES TESTS SANGUINS DANS LES ACTIONS EN RECHERCHE DE PATERNITÉ ......... 233

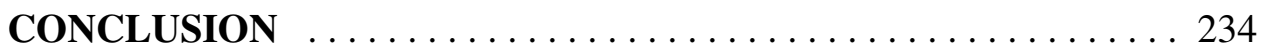




\section{INTRODUCTION}

Il y a quelques siècles, le droit était une discipline parfaitement autonome et le tribunal pouvait prendre des décisions à la seule lumière de ses connaissances juridiques. Le développement exceptionnel de la science et de la technologie a entraîné depuis un siècle des situations de plus en plus complexes, rendant discutable, voire même impossible sans l'éclairage des experts, la résolution de nombreux litiges sur la seule base du droit. Progressivement, les tribunaux n'ont eu d'autres choix que de demander l'assistance de spécialistes pour mieux comprendre et prendre la meilleure décision dans la solution d'un litige. C'est ainsi que l'expert a fait son entrée dans l'enceinte du tribunal. À l'origine, les tribunaux le considéraient plutôt avec une certaine méfiance. En effet, les juges le percevaient comme une atteinte à leur capacité d'appréciation du litige et craignaient de voir leurs pouvoirs usurpés par les spécialistes. C'était l'époque du tâtonnement où l'on situait mal le rôle de l'expert en croyant erronément qu'il constituait une atteinte au pouvoir discrétionnaire des tribunaux. Malgré cet état d'esprit peu favorable, la magistrature a constaté que les experts pouvaient lui apporter une aide précieuse qu'elle ne pouvait plus négliger et, disons-le, qu'elle apprécie de plus en plus, dans les litiges où la compréhension des faits et de leur portée dépend de l'expertise, ce qui permet de prendre une décision plus éclairée.

Depuis de nombreuses années, nous avons assisté à une augmentation des circonstances qui nécessitent l'intervention d'un expert, d'où la multiplication non seulement du nombre, mais également de la nature des expertises qui sont devenues complexes par la spécialisation de plus en plus poussée. Nous sommes confrontés à une science sans cesse «plus savante» et à une technologie sophistiquée rendant parfois difficile, même pour l'initié, la maîtrise de toutes les subtilités d'une situation. Le contrôle du litige semble, pour ce motif, échapper un peu au tribunal qui peut hésiter parfois à contredire un scientifique hautement qualifié. Nous sommes arrivés à une étape où le droit ne peut plus ignorer les progrès qui surviennent dans les autres disciplines et les tribunaux, qui doivent chercher à rendre une justice toujours meilleure, ont cherché dans cette perspective à trouver des moyens et des critères pour situer le rôle de l'expert et limiter l'influence de l'expertise aux objectifs poursuivis lorsqu'on la requiert. 
La présence de l'expert dans les cours de justice a donc amené les tribunaux à définir son rôle en le considérant comme un conseiller capable de fournir au juge un outil supplémentaire, l'expertise, pour l'aider à solutionner le litige. Quel que soit le niveau de connaissance du juge, il ne peut embrasser toutes les sciences, maîtriser toutes les techniques, pénétrer la pensée de chacun. Pourtant, toutes ces connaissances sont souvent nécessaires pour prendre une décision juste et éclairée. Le rôle de l'expert consistera justement à apporter une aide aussi objective que possible afin que le tribunal puisse tirer ses propres conclusions en appliquant avec justesse les données scientifiques et technologiques aux faits mis en preuve devant lui.

En droit civil général, et plus particulièrement en matière familiale, l'expert, à partir de la base factuelle du litige, donnera un avis qui nécessairement repose sur une évaluation comportant des éléments subjectifs. À titre d'exemples, il en sera ainsi lorsqu'il s'agit de déterminer lequel des deux parents est le plus apte à se voir confier la garde d'un enfant; quel régime de protection serait le mieux adapté pour le majeur inapte et si sa garde en établissement est nécessaire; quelles sont les probabilités qu'un enfant ait été victime d'abus physiques ou sexuels; si le degré d'autonomie d'une personne et ses aptitudes sont suffisants pour lui permettre de réintégrer le marché du travail à la suite d'un divorce; si la reprise en charge d'un enfant est possible en matière d'adoption ou de garde; si le niveau de la capacité mentale d'une personne lui permet de consentir à des mesures volontaires en matière de protection de la jeunesse. Ces quelques illustrations montrent que l'expertise peut couvrir une multitude de situations que le tribunal doit trancher.

L'intérêt de l'étude de l'expertise en matière familiale apparaît lorsqu'on s'arrête à l'impact qu'elle peut avoir sur les personnes concernées par le litige. L'évolution des diverses théories et conceptions scientifiques a amené des expertises contradictoires, sans compter qu'elles peuvent aussi résulter du fait que les experts ne retiennent pas les mêmes éléments, ne partent pas des mêmes prémisses, ne considèrent pas tous les faits ou ne rencontrent pas toutes les personnes intéressées. Comment les tribunaux doivent-ils réagir en présence de ces expertises contradictoires? Quelle portée doivent-ils donner à une expertise scientifique faisant état, par exemple, de $90 \%$ de certitude alors qu'ils doivent décider selon la prépondérance de la preuve? 
Nous ne croyons pas nécessaire d'analyser, de façon exhaustive, toutes les situations pouvant donner lieu à une expertise. Il nous apparaît plus opportun d'analyser les différentes règles qui encadrent l'avis du scientifique auquel on demande d'éclairer le tribunal sur des situations très variables.

Nos recherches montrent qu'il n'y a pas de distinctions significatives justifiant de traiter séparément l'expertise en matière civile de celle en matière familiale, sous réserve de certaines différences dont nous ferons état, le cas échéant. En effet, nous verrons que le rôle de l'expert, et toutes les règles générales concernant l'expertise et la contre-expertise, obéissent aux mêmes principes et souvent aux mêmes contraintes.

Dans cette perspective, nous allons aborder dans la première partie le cadre général de l'expertise, en traitant plus particulièrement du droit à l'expertise, de certaines catégories d'expertises et des limites de l'expertise.

Nous étudierons, dans la deuxième partie, l'influence de l'expertise sur l'adjudication d'un litige en analysant les différences entre la recevabilité et l'admissibilité de la preuve par expert.

Finalement, à la lumière du droit comparé nous formulerons, dans la troisième partie, certaines critiques et suggestions concernant la preuve par expert en droit québécois, afin d'utiliser plus efficacement l'expertise au sein d'un litige civil.

\section{PREMIÈRE PARTIE : LE CADRE GÉNÉRAL DE L'EXPERTISE}

L'impact considérable de l'expertise justifie à lui seul l'analyse des répercussions de ce moyen de preuve dans l'administration de la justice qui peut, tantôt être contraint par le secret professionnel, tantôt par l'inviolabilité physique et psychologique d'une personne. De plus, même si l'on poursuit l'objectif de rendre une plus grande justice, l'expertise touchant la personne ne doit pas cependant avoir recours à des méthodes abusives. Il faut donc se demander s'il est possible de concilier la protection de la personne avec les divulgations que peuvent fournir les experts. 
L'expertise constitue le point de rencontre entre le droit et les autres disciplines qui contribuent à la recherche de la vérité et d'une meilleure justice. Le droit et les sciences propres aux diverses sortes d'expertises obéissent à des règles distinctes, ce qui provoque, à l'occasion, des confrontations de valeurs.

\section{CHAPITRE I : LE DROIT À L'EXPERTISE}

L'un des aspects les plus discutés en matière d'expertise concerne souvent la confrontation du principe de l'inviolabilité de la personne humaine avec le droit à l'expertise. S'il existe des exceptions au principe de l'inviolabilité de la personne, il reste à répondre, au-delà de ces exceptions, à l'épineuse question de déterminer quelle portée peut avoir le refus d'une personne à se soumettre à une évaluation alors qu'aucune raison ne pourrait, à première vue, justifier un tel refus. Comment équilibrer le «refus» et le droit à la meilleure preuve? L'expertise portant sur une partie qui en a fait la demande permet-elle à l'autre de requérir une contre-expertise?

\section{Section 1 : L'intérêt requis pour demander une expertise et une contre- expertise}

On doit avoir un intérêt dans le litige pour être admis à demander une expertise ou une contre-expertise. À ce titre on retrouve, parmi les premiers intéressés, les parties au procès qui peuvent avoir recours à un expert pour établir leurs prétentions ${ }^{1}$. Chaque partie peut faire appel à l'expert de son choix, ce qui entraîne fréquemment plusieurs expertises dont les conclusions sont irréconciliables $^{2}$. En matière familiale, pour pallier cet inconvénient, il est

1. Par exemple, un père soupçonné d'avoir abusé sexuellement de son enfant peut assigner plusieurs médecins, à titre d'experts, afin de réfuter ces allégations, voir Protection de la jeunesse-402, J.E. 89-902 (C.Q.).

2. Louis GÉLINAS et Bartha Maria KNOPPERS, «Le rôle des experts en droit québécois en matière de garde, d'accès et de protection», (1993) 53 R. du B. 3, 17. Dans l'arrêt Droit de la famille-1242, [1989] R.D.F. 287 (C.S.), la requérante demande au tribunal de lui confier la garde de son enfant. Pour appuyer ses prétentions, chaque partie a fait entendre un témoin expert. L'expert de la requérante conclut qu'il est dans l'intérêt de l'enfant de retourner vivre avec sa mère. La psychologue retenue par le père conclut, pour sa part, que l'enfant est très bien avec son père. Devant une telle preuve, le tribunal s'est appuyé sur la preuve profane pour rejeter les conclusions de l'expert de la requérante. Voir également, Droit de la famille251, [1986] R.D.F.11 (C.S.). 
possible, lorsque les parents collaborent, de ne consulter qu'un seul expert pour évaluer la situation de l'ensemble de la famille ${ }^{3}$.

Le tribunal peut aussi avoir un intérêt pour demander une expertise ${ }^{4}$. En effet, les articles 414 et suivants du Code de procédure civile $e^{5}$ accordent au tribunal le pouvoir d'ordonner une expertise lorsque les fins de la justice le requièrent ${ }^{6}$. Il en est ainsi, à titre d'exemple, lorsque le juge, à cause de la technicité des faits du litige, ne peut maîtriser tous les aspects du dossier ${ }^{7}$ ou encore si les expertises présentées s'avèrent contradictoires et qu'il désire obtenir l'avis d'un expert indépendant des parties et probablement plus impartial ${ }^{8}$.

3. C'est ce qui s'est produit, entre autres, dans les affaires suivantes : X. c. Z., [1975] C.S. 508. Il s'agissait d'une contestation au sujet de la garde des enfants. Les parties ont accepté de se soumettre à une évaluation conjointe afin d'aider le tribunal à prendre une décision éclairée; C.B. c. F.M., [1988] 11 Q.A.C. 229. Dans cette affaire, les parties ont consenti à ne consulter qu'un seul expert afin de ne pas allonger les délais indûment. Il s'agissait d'un litige sur la garde des enfants.

4. L. GÉLINAS et B.M. KNOPPERS, loc. cit., note 2, voir p. 18 en matière de garde et p. 29 en matière de protection.

5. L.R.Q., c. C-25.

6. En effet, même en matière familiale le tribunal détient, dans certaines situations, le pouvoir de contraindre quelqu'un à se soumettre à une expertise. Ainsi, dans Droit de la famille-628, [1989] R.D.F. 321 (C.A.), où il s'agissait de déterminer à quel parent serait confiée la garde des enfants, le tribunal a fait droit à la requête du père demandant que les enfants se soumettent à une évaluation psychologique. Le tribunal motive sa décision en déclarant que l'état psychologique des enfants est au coeur même du litige dont il constitue d'ailleurs le principal objet; dans Droit de la famille-1381, [1991] R.D.F. 130 (C.S.) le tribunal a ordonné une expertise psychosociale des parties afin de déterminer les droits de visite et de sortie.

7. Voir, entre autres, l'affaire Donolo inc. c. St-Michel Realties Inc., [1971] C.A. 536, 537, où la Cour d'appel s'exprime ainsi sur la légitimité pour le juge de première instance d'avoir nommé ses propres experts : «[...] dans leur recherche de la solution des problèmes qui leur sont ainsi soumis, les juges sont justifiés de se renseigner et de se faire éclairer par ceux qui ont plus particulièrement consacré leurs activités professionnelles, soit à acquérir une connaissance approfondie de ces sciences et de la technique de leurs applications, soit à acquérir de l'expérience dans le domaine de cette technique ou de ces applications».

8. Jean-Claude ROYER, La preuve civile, Cowansville, Éditions Yvon Blais inc., 1987, $\mathrm{n}^{\circ} 485$, p. 178; Henri KÉLADA, Notions et techniques de la preuve civile, Montréal, Wilson et Lafleur ltée, 1986, p. 273. 
Le pouvoir du tribunal de recourir à un expert est également prévu par les Règles de pratique de la Cour supérieure en matière familiale 9 qui prévoient que le juge peut suggérer aux parents de se soumettre à une expertise. Le tribunal peut s'appuyer sur ces dispositions en matière de garde et de droit de visite lors d'un divorce ou d'une séparation ${ }^{10}$.

Dans les situations où la Loi sur la protection de la jeunesse ${ }^{11}$ s'applique, l'article 86 confère au directeur de la protection de la jeunesse le pouvoir de demander qu'un expert procède à l'évaluation psychologique du milieu familial de l'enfant ${ }^{12}$. Il s'agit d'un pouvoir discrétionnaire, sauf l'obligation de procéder à une expertise lorsque le tribunal lui en fait la demande ${ }^{13}$.

En dernier lieu, dans tous les litiges où son bien-être est mis en question, l'enfant possède l'intérêt nécessaire pour requérir une expertise. À cette fin, on lui nomme un représentant, en vertu de l'article 394.1 C.p.c. ou des articles 80 et 84 de la Loi sur la protection de la jeunesse. Le rôle de ce représentant est de protéger les intérêts de l'enfant lorsque ceux-ci sont opposés à ceux de ses parents ${ }^{14}$. Pour le représenter adéquatement, il peut demander une expertise pour contredire celles déjà produites lorsqu'il estime qu'elles ne prennent pas en considération les besoins de l'enfant.

9. L.R.Q., c. C-25, r. 9, art. 23 à 24. Voir, entre autres, Monique OUELLETTE, Droit de la famille, $2^{\mathrm{e}}$ éd., Montréal, Éditions Thémis, 1991, p. 351 et Claude C. BOULANGER, Divorce, $2^{\mathrm{e}}$ éd., vol. 202, Montréal, Wilson et Lafleur ltée, 1990, pp. 31-32.

$10 . \quad$ L. GÉLINAS et B.M. KNOPPERS, loc. cit., note 2, 18-20.

11. L.R.Q., c. P-34.1, ci-après citée L.P.J.

12. Voir, à ce sujet, Protection de la jeunesse-339, J.E. 88-883 (T.J.), où le tribunal se prononce sur le droit du directeur de la protection de la jeunesse de demander une expertise en vertu de l'article 86 de la Loi sur la protection de la jeunesse. Voir aussi, Protection de la jeunesse-572, [1992] R.J.Q. 2354 (C.Q.).

13. L.P.J., art. 86(2).

14. Dans Protection de la jeunesse-635, [1993] R.D.F. 451 (C.Q.), les enfants désiraient se faire adopter par leur famille d'accueil. Un avocat les représentait afin de protéger leurs intérêts qui étaient opposés à ceux de leur mère. Voir également, Protection de la jeunesse-572, précité, note 12; Suzanne GUILLET, «Le sort réservé aux enfants à l'occasion d'un litige conjugal», dans Droit civil et procédure civile, vol. 2, Cowansville, Éditions Yvon Blais inc., p. 54 , aux pages 73 et 74 . 


\section{Section 2 : La personne contraignable à l'expertise}

L'expertise peut être demandée, comme nous venons de le voir, par différentes personnes. Cependant, le pouvoir de demander n'implique pas nécessairement celui de contraindre. En principe, personne n'est obligé de consentir à une évaluation le concernant, mais il existe des situations qui dérogent à ce principe.

\section{A) Le principe : l'absence d'obligation de se soumettre à l'expertise}

Dans notre droit, l'intégrité physique et psychologique d'une personne est protégée par la Charte québécoise qui énonce le principe de l'inviolabilité de la personne humaine. En effet, nul ne peut être forcé de se soumettre à une expertise sans y avoir consenti ${ }^{15}$. Toutefois, cette règle s'applique seulement lorsque l'expertise implique la violation de l'intégrité physique ou psychologique d'une personne. À l'inverse, si l'étude ne porte pas sur un individu, comme en matière de bornage, l'expertise peut être légalement imposée ${ }^{16}$.

Les litiges sur la filiation soulèvent fréquemment le problème du consentement à l'expertise sanguine. La jurisprudence a adopté, à ce propos, une position non équivoque selon laquelle, en l'absence d'une disposition à l'effet contraire, le tribunal doit respecter le principe de l'inviolabilité de la personne humaine : il ne peut que suggérer un test sanguin ${ }^{17}$. Cette restriction relative à l'expertise sanguine s'applique également aux autres situations qui touchent à l'intégrité physique ou psychologique d'un individu.

La jurisprudence semble moins unanime quant aux effets du refus de consentir à l'expertise. Certains prétendent que le refus constitue l'exercice d'un droit fondamental et qu'aucune présomption négative ne peut en être tirée ${ }^{18}$.

15. C.c.Q., art. 10; Charte des droits et libertés de la personne, L.R.Q., c. C-12, art. 9.

16. Léo DUCHARME, L'administration de la preuve, Montréal, Wilson et Lafleur ltée, 1986, $\mathrm{n}^{\mathrm{o}}$ 603, p. 199.

17. Dans Droit de la famille-1859, [1993] R.J.Q. 2303, 2309 (C.A.), le tribunal déclare qu'il ne peut que suggérer un prélèvement de sang étant donné l'absence d'une disposition législative l'autorisant à l'imposer.

18. Le tribunal, dans l'arrêt Droit de la famille-1111, [1987] R.D.F. 331 (C.S.), déclare à la page $332: \ll[\ldots]$ que le refus d'un défendeur de se soumettre à une analyse sanguine, considéré dans l'affaire précitée de Droit de la famille-1059 comme un indice additionnel pour rendre 
Cependant, une nouvelle tendance semble voir le jour suivant laquelle le juge pourrait interpréter négativement le refus ${ }^{19}$. Pour notre part, nous croyons justifiés les propos d'Albert Mayrand, qui a écrit :

«[...] Par contre, on ne doit pas se servir du principe de l'inviolabilité de la personne humaine comme d'un paravent pour cacher la vérité. Si on refuse sans motif raisonnable de se prêter à un acte médical qui ne présente aucun danger et aucun inconvénient sérieux, c'est probablement parce que l'expertise doit révéler un fait que l'on a intérêt à cacher. Il est donc logique d'interpréter contre le récalcitrant son refus de collaborer».

«[...] On ne doit pas pousser jusqu'à l'absurde l'application du principe de l'inviolabilité de la personne; on ne demande pas à un plaideur de se laisser saigner à blanc, mais de se prêter à un prélèvement indolore. La justice et la vérité valent bien une goutte de sang» ${ }^{20}$.

admissible la preuve testimoniale, ne saurait avoir, même uniquement, cette portée d'être défavorable, car il ne prouve rien et est l'exercice d'un droit par le défendeur». Voir également l'opinion du juge Proulx dans Droit de la famille-1059, [1990] R.D.F. 385 (C.A.), 392.

19. Droit de la famille-1859, précité, note 17, 2310. Il s'agit d'une action en déclaration de paternité où le défendeur a refusé de se soumettre à un test sanguin sans justifier son refus. Quant à l'impact à donner à ce refus, le tribunal s'exprime ainsi : «Étant acquis qu'un tribunal ne peut que suggérer un prélèvement sanguin, notre cour a aussi conclu qu'en cas de refus injustifié le tribunal peut tirer de ce refus une conclusion qui sera généralement négative à l'égard de celui qui s'y refuse [...]» et il ajoute à la page 2311 : «Et ce n'est pas, à mon avis, une hérésie que de tirer une inférence du refus de se soumettre à un test sanguin alors qu'il y a un intérêt social réel à ce qu'un enfant connaisse l'identité de son père [...]». Aux pages 2311 et suivantes, on fait état des nombreuses provinces canadiennes où le tribunal peut tirer les conclusions qu'il estime indiquées d'un refus de se soumettre à un tel test. L'opinion du juge Vallerand dans Droit de la famille-1059, précité, note 18, va dans le même sens. Voir également Droit de la famille - 1185, [1988] R.D.F. 260 (C.S.), où le tribunal a tiré une impression défavorable du refus de la mère de se soumettre aux évaluations réalisées par l'expert du père. Le tribunal avait d'abord obtenu le consentement des parties afin qu'un expert du Centre d'expertise psychosociale procède à l'évaluation de la famille. Par la suite, les deux parties avaient demandé la réalisation d'expertises privées.

20. Albert MAYRAND, L'inviolabilité de la personne humaine, Montréal, Wilson et Lafleur ltée, 1975, pp. 106-107. 
En France, une disposition du Code de procédure civile ${ }^{21}$ autorise le juge à considérer comme établis les faits que l'expertise avait pour but de constater. Il serait souhaitable qu'une telle disposition soit adoptée au Québec. Spécialement en matière familiale lorsqu'on doit déterminer l'intérêt d'un enfant. Nous croyons qu'un juge, ne pouvant bénéficier d'un élément aussi essentiel qu'une expertise à cause d'un refus injustifié, devrait pouvoir logiquement en inférer une présomption défavorable même si en théorie cela va à l'encontre du principe de l'inviolabilité de la personne.

En terminant sur le consentement à l'expertise, signalons qu'en matière de protection de la jeunesse, le droit de refuser une évaluation est prévu à l'article 87 L.P.J., où l'on édicte que l'enfant âgé de 14 ans et plus et les parents peuvent refuser de s'y soumettre ${ }^{22}$, leur consentement étant indépendant l'un de l'autre. Pour l'enfant âgé de moins de 14 ans, ce sont ses parents qui prennent la décision pour lui.

\section{B) Les exceptions à l'«incontraignabilité» de l'expertise}

Le législateur et les tribunaux ont tempéré le principe du droit de consentir à l'expertise lorsqu'un intérêt supérieur l'exigeait. On peut identifier deux catégories d'exceptions, soit celles relatives à l'inviolabilité de la personne et celles justifiées par le droit de contredire une expertise.

\section{Les exceptions au principe de l'inviolabilité de la personne humaine}

Les exceptions au principe général de l'inviolabilité de la personne humaine relèvent de deux sources. Il s'agit de l'expertise imposée par la loi et du pouvoir du tribunal et du directeur de la protection de la jeunesse de requérir une expertise.

21. Code de procédure civile français, art. $11:$ :Les parties sont tenues d'apporter leur concours aux mesures d'instruction sauf au juge à tirer toute conséquence d'une abstention ou d'un refus».

22. Voir, à ce sujet, Renée JOYAL, Précis de droit des jeunes, t. 2, Cowansville, Éditions Yvon Blais inc., 1986, $\mathrm{n}^{\circ} 86$, p. 48. 


\section{a) L'expertise imposée par la loi}

On relève, dans la législation, certaines situations où une personne peut être contrainte légalement à subir une expertise. Il en est ainsi, par exemple, lorsqu'il s'agit d'appliquer les articles 399 et 400 C.p.c. ${ }^{22 a}$ La formulation de l'article 399 C.p.c. le rend applicable à un grand nombre de situations. Il n'est donc pas surprenant de constater que cet article a son utilité en matière de séparation et de divorce ${ }^{23}$. En effet, l'état de santé physique ou mental d'une partie peut être déterminant dans un litige concernant le montant de la pension alimentaire ou la garde des enfants ${ }^{24}$.

Il en sera de même lorsqu'il existe des motifs sérieux de croire qu'une personne représente un danger pour elle-même ou pour les autres en raison de l'état de sa santé mentale. Avant de conclure à la nécessité de la garder en

22.a. À titre complémentaire, consulter, J.-C. ROYER, op. cit., note 8, nos 606-614, pp. 222-225. L'article 399 C.p.c. édicte que : «Dans toute cause susceptible d'appel, lorsqu'est mis en question l'état physique ou mental d'une personne, partie à un litige ou qui a subi le préjudice qui y a donné lieu, une partie peut assigner à ses frais cette personne par bref de subpoena, pour qu'elle se soumette à un examen médical». On ne peut pas s'objecter à une requête fondée sur l'article 399 C.p.c., en invoquant le principe de l'inviolabilité de la personne humaine. Dans Syndicat des travailleurs(euses) de l'enseignement Chauveau-Charlesbourg c. Robitaille-Rousseau, [1988] R.D.J. 259, 266 (C.A.), on a rejeté l'argument basé sur la Charte québécoise des droits et libertés qui protège le droit à l'intégrité et à la liberté de sa personne. L'enfant qui souffrait de trisomie 21 voulait être admis en classe régulière. Le tribunal a jugé qu'en voulant se faire reconnaître un droit, l'argument fondé sur la Charte n'était pas utile en l'instance. Voir aussi Stedman c. Cordier, 1976. R.P. 372, 373 (C.S.). Toutefois, cette exception comporte une limite : on devrait refuser d'obliger une partie à se soumettre à des examens douloureux ou indûment prolongés : A. MAYRAND, op. cit., note 20, p. 109. L'auteur énumère certains examens médicaux qui constituent, selon lui, la limite de ce qu'on peut imposer à une partie. Il mentionne, entre autres, l'examen radiographique après ingestion de barium et le prélèvement de l'estomac au moyen d'un tube de caoutchouc.

23. Stedman c. Cordier, précité, note 22a, 373, où le tribunal s'exprime ainsi concernant l'application de l'article 399 C.p.c. au cas de divorce et de séparation : «Après réflexion, le tribunal croit que l'article 399 permet de faire examiner la partie adverse dans toute action qui met en question la santé physique ou mentale d'une partie, y compris les cas de divorce et de séparation.».

24. Dans Rousseau c. Lemay-Rousseau, [1976] C.A. 317, il s'agissait d'un litige portant sur le montant de la pension alimentaire. L'appelant a demandé que l'intimée se soumette à un examen médical en vue de déterminer si elle était apte à subvenir à ses besoins. La cour fut d'avis qu'il avait le droit d'exiger un tel examen. 
établissement il faut obligatoirement la soumettre à une évaluation psychiatrique $^{25}$.

Il existe aussi d'autres lois qui assujettisent l'exercice d'un droit à un examen médical ou psychologique. À titre d'exemple, on peut consulter la Loi sur l'assurance automobile ${ }^{26}$ et la Loi sur les accidents du travail et les maladies professionnelles ${ }^{27}$ qui posent une telle condition. On peut, d'ailleurs, s'interroger sur les motifs qui ont pu inciter le législateur à refuser de rendre obligatoire l'expertise sanguine qui concerne une question d'ordre public, la filiation d'un enfant. L'expertise sanguine est tellement supérieure aux témoignages contradictoires $^{28}$ qu'il est difficile de comprendre qu'on ait pu priver l'enfant ou le père de recourir à ce moyen de preuve compte tenu des dérogations déjà acceptées à l'inviolabilité de la personne humaine.

\section{b) Le pouvoir du tribunal et du directeur de la protection de la jeunesse en matière d'expertise}

Lorsque l'on traite d'expertise sur la personne, le tribunal peut en de très rares occasions faire échec au principe de l'inviolabilité de la personne humaine. Qui plus est, il ne peut le faire que si une disposition législative le prévoit ${ }^{29}$. Ainsi, en matière familiale, les articles 23 à 24 des Règles de pratique en matière familiale $e^{30}$ énoncent que le juge ne rend une ordonnance d'expertise psychosociale que du consentement des parties ${ }^{31}$. Malgré cela, il semblerait que

25. C.c.Q., art. 26 et suivants. Loi sur la protection du malade mental, L.R.Q., c. P-41.

26. L.R.Q., c. A-25.

27. L.R.Q., c. A-3.001. Voir également : Loi sur la protection de la santé publique, L.R.Q., c. P-35; Loi sur les adoptions d'enfants domiciliés en République populaire de Chine, L.R.Q., c. A-7.01.

28. Effectivement, l'expertise sanguine visant à identifier l'ADN est un test dont la précision est de $99.8 \%$ lorsqu'il s'agit de déterminer la filiation d'une personne. «Le test d'ADN est de plus en plus utilisé pour déterminer la paternité», La Presse, Montréal, le 18 février 1994.

29. L. DUCHARME, op. cit., note $16, \mathrm{n}^{\circ} 525$, p. 175 : «Le principe de l'inviolabilité de la personne humaine s'oppose à ce qu'on puisse soumettre une personne à un examen médical contre sa volonté. Seule une disposition expresse de la loi peut déroger à cette règle». Plusieurs juges ont réitéré cette affirmation pour refuser d'obliger quelqu'un à se soumettre à une expertise. Voir, entre autres, Droit de la famille -206, [1986] R.J.Q. 2038, 2042 (C.A.); Droit de la famille-1859, précité, note 17.

30. Ci-après nommée «R.p.C.s. mat. fam.».

31. R.p.C.s. mat. fam., art. 23.2. 
l'article 414 du Code de procédure civile pourrait servir de fondement au pouvoir du tribunal d'enjoindre la famille à se soumettre à une évaluation psychosociale. Cet article édicte que :

«Art. 414 Après contestation liée, le tribunal peut, même de sa propre initiative, s'il est d'avis que les fins de la justice peuvent être ainsi mieux servies :

1. ordonner une expertise par personne qualifiée, qu'il désigne, pour l'examen, la constatation et l'appréciation de faits relatifs au litige;

\section{2. $[\ldots] \gg$.}

Toutefois, dans le contexte de la Loi sur la protection de la jeunesse, le tribunal ne pourrait pas recourir à l'article 414 C.p.c. En effet, ce dernier n'y trouverait pas d'application ${ }^{32}$ puisqu'on prévoit expressément les cas où l'expertise est obligatoire.

Dans les cas relevant de la Chambre de la jeunesse, la situation est claire. Il n'y a que deux situations où la demande d'une expertise par le tribunal ou par le directeur de la protection de la jeunesse est obligatoire, et c'est lorsqu'on soupçonne que l'enfant a été victime de mauvais traitements ou d'abus sexuels ${ }^{33}$.

\section{Le droit à la contre-expertise}

Le droit de contredire une expertise est consacré par la règle audi alteram partem qui énonce le droit de toute partie à un litige d'être entendue et de courant jurisprudentiel soutient que l'article 85 L.P.J. n'a pas pour effet d'exclure de son application les articles du Code de procédure qui n'y sont pas énumérés. Infra., note 125.

33. Voir art. 38(g) et 87 L.P.J.; Protection de la jeunesse-339, précité, note 12, 5, où le tribunal, après avoir traité du pouvoir du tribunal et du directeur de la protection de la jeunesse de requérir une expertise et de l'obligation de s'y soumettre dans les cas visés à l'article $38(\mathrm{~g})$, conclut ainsi : «Donc, la situation est claire ici puisque c'est pour ce motif (abus sexuel) que le jugement de déclaration de compromission a été prononcé. Le parent ne peut refuser l'évaluation psychologique, que ce soit à la demande discrétionnaire du Directeur de la protection de la jeunesse ou moins encore lorsqu'il y a un ordre du Tribunal». (Les parenthèses et le contenu dans la note 33 sont de nous.) 
répondre utilement aux prétentions de la partie adverse ${ }^{34}$. Ainsi, lorsqu'une partie s'est soumise à une première évaluation, les tribunaux n'hésitent pas à passer outre le principe de l'inviolabilité de la personne humaine afin de l'obliger à en subir une seconde. En effet, la partie adverse doit avoir la possibilité de présenter une contre-expertise comparable à la précédente afin d'attaquer efficacement le bien-fondé des conclusions auxquelles elle arrive.

\section{CHAPITRE II : CERTAINES CATÉGORIES D'EXPERTISE}

Nous allons concentrer ce chapitre sur les expertises comportant des données subjectives. En effet, les expertises en matière familiale portent le plus souvent sur des rapports entre les personnes où les éléments psychologiques ou émotifs sont dominants. Ces types d'expertises nécessitent que l'expert apprécie une situation afin de faire ensuite des recommandations au tribunal, selon le meilleur intérêt de l'enfant, par exemple, lorsque celui-ci fait l'objet d'une dispute entre les parents.

\section{Section 1: L'expertise portant sur des faits révélés en cour}

L'article 414 C.p.c. donne au juge pleine discrétion, à la demande des parties et même de sa propre initiative, d'ordonner «une expertise par personne qualifiée, qu'il désigne, pour l'examen, la constatation et l'appréciation de faits relatifs au litige». Ces experts devront dans certains cas, comme l'indique le texte de l'article 414 C.p.c., assister à tout le procès ${ }^{35}$.

34. Dans Protection de la jeunesse-432, J.E. 90-446 (C.Q.), p. 7, le tribunal s'exprime ainsi à propos de la règle audi alteram partem : «En vertu de la règle «audi alteram partem», chaque partie doit pouvoir répondre de façon utile et efficace aux prétentions des autres parties. Lorsqu'il admet un élément de preuve en provenance d'une partie, le Tribunal doit permettre aux autres parties de contredire. Le Tribunal doit offrir à toutes les parties une égale opportunité de faire entendre leurs témoins». Dans Guimond c. Morin, J.E. 94-107 (C.A.), la Cour d'appel a renversé le jugement de la Cour supérieure pour le motif qu'en refusant à une partie le droit de faire entendre ses témoins, elle avait ainsi enfreint la règle audi alteram partem.

35. Voir, à ce sujet, Hôtel-Dieu de Québec c. Bois, 1977] C.A. 563, 568, dans laquelle la Cour d'appel déclare : «Quant aux experts d'opinion, ils se doivent d'assister aux séances de la Cour pour pouvoir commenter sur les faits relatés à l'audience et, dans un cas comme le présent, pour apprécier le témoignage des défendeurs au point de vue clinique, pour approuver ou désapprouver les traitements des médecins, exposer au juge les techniques de la science médicale, et conclure que les défendeurs se sont ou ne se sont pas conformés aux 
Il est préférable, malgré l'article 82 L.P.J. traitant du huis clos, de permettre à un expert de demeurer présent dans la salle d'audience pour aider le tribunal tant dans la recherche des faits que dans l'éventuelle imposition de mesures à prendre pour pallier une situation. Néanmoins, dans sa discrétion, le juge peut ordonner l'exclusion des témoins experts afin de permettre aux témoins de s'exprimer plus facilement, ce qui n'est pas toujours le cas lorsqu'un expert est présent $^{36}$.

Eu égard à ce qui précède, l'expert pourra se prononcer sur les témoignages des témoins ordinaires et ceux des témoins experts ${ }^{37}$. De même, il peut être amené à donner son interprétation des témoignages des autres témoins experts ou encore sur le rapport que ceux-ci ont préparé dans le cadre d'une instance ${ }^{38}$.

\section{Section 2: L'expertise fondée sur les données recueillies hors cour}

Il ne sera possible pour l'expert, dans certaines hypothèses, de se prononcer que s'il a préalablement rencontré les parties. Il en est ainsi, par exemple, d'un litige sur la garde : il ne peut se faire une opinion et formuler ses recommandations sans avoir rencontré les personnes impliquées ${ }^{39}$. Examinons quelques

règles de l'art; toutes des opinions que le juge n'est pas obligé d'accepter mais qui l'éclaireront dans le jugement qu'il aura à rendre». Le tribunal réitéra le même principe dans Protection de la jeunesse-567, [1992] R.D.F. 730, 731 (C.Q.).

36. Protection de la jeunesse-642, J.E. 93-1599 (C.Q.).

37. Le jugement dans Protection de la jeunesse-572, précité, note 12, démontre clairement l'application pratique de ce principe. Il s'agissait d'une affaire visant à obtenir une ordonnance de placement pour deux jeunes enfants. Le psychologue fut entendu en premier lieu devant le tribunal, et par la suite à la toute fin après que les quelque onze témoins eurent témoigné. Il a alors constaté : «[...] l'existence d'un certain potentiel conflictuel, mais il a été agréablement impressionné, et à certains égards rassuré, par les propos tenus et les comportements observés».

38. $\quad$ Protection de la jeunesse-605, [1993] R.J.Q. 739 (C.S.).

39. Il en est de même lorsqu'il s'agit de déterminer la capacité de consentir à des traitements médicaux : Douglas Hospital Center c. T. (C.), [1993] R.J.Q. 1128 (C.S.); Hôpital StCharles-Borromée c. G. (G.), J.E. 94-106 (C.S.) ; ou de les refuser, de se prononcer sur la nécessité de la garde en établissement d'une personne : Supra, Chapitre I de la Partie I, sous le titre : «L'expertise imposée par la loi»; d'évaluer les aptitudes à retourner au travail lors d'une requête en modification de pension alimentaire entre ex-conjoints : voir Droit de la famille-1701, J.E. 93-18 (C.A.), où lors d'un divorce, en 1987, l'intimée avait 47 ans et n'avait jamais travaillé durant le mariage qui a duré 23 ans. Elle voulut prouver son état persistant de dépendance financière et son incapacité de réintégrer le marché du travail; ou entre ascendants et descendants, lorsque le créancier alimentaire soutient ne pouvoir 
situations où il est fréquent de faire appel à des experts afin d'illustrer la variété des expertises fondées sur des données recueillies hors cours.

\section{A. Les tests d'évaluation psychologique}

\section{L'évaluation des capacités intellectuelles}

L'équilibre psychologique et la stabilité sont essentiels chez le parent qui assume la garde d'un enfant ${ }^{40}$. Dans Droit de la famille- $653^{41}$, l'expert affirmait que la mère schizophrène, n'ayant jamais vu son enfant puisqu'il est en famille d'accueil depuis sa naissance, ne pourrait jamais être considérée comme une mère ayant les aptitudes pour prendre en charge un enfant. De plus, les gestes et l'attitude d'un parent ayant pour but d'empêcher des contacts avec l'autre parent peuvent justifier qu'on lui enlève la garde s'il y va du meilleur intérêt de l'enfant $^{42}$. L'évaluation des facultés intellectuelles s'avère donc importante.

Il en est de même en matière d'adoption lors d'une demande de rétractation du consentement, alors que le tribunal devra déterminer si le parent était

subvenir à ses besoins : Droit de la famille-1618, J.E. 94-176 (C.A.). Ces exemples montrent l'étendue du domaine de l'expertise.

40. Droit de la famille-1242, précité, note 2. Dans Droit de la famille-1717, [1993] R.J.Q. 166 (C.S.), la preuve démontrait que l'intimée était atteinte du syndrome d'aliénation parentale, car elle avait réussi à empêcher ou réduire presque à néant les rapports entre le père et sa fille en dénonçant à des médecins et des policiers que le requérant avait agressé physiquement et sexuellement sa fille. Les tribunaux québécois ont reconnu ce syndrome. Voir par exemple, Droit de la famille-1485, [1991] R.D.F. 570, 574-575 (C.S.); Droit de la famille1549, [1992] R.D.F. 316 (C.A.); Droit de la famille-1385, [1991] R.D.F. 180 (C.S.); E.H. c. L.V., C.S. Montréal, $\mathrm{n}^{\circ}$ 500-12-159976-871, le $1^{\text {er }}$ novembre 1990, dans Jean-François BOULAIS, Loi sur la protection de la jeunesse, texte annoté, $2^{\mathrm{e}}$ éd., Montréal, Société québécoise d'information juridique, 1990, à la page 18, où le juge s'est estimé justifié de suspendre l'exercice de tous les privilèges de l'autorité parentale du père en concluant qu'un parent capable d'utiliser une personne aussi vulnérable qu'un enfant pour assouvir ses désirs de vengeance à l'endroit de son ex-conjoint n'est pas en mesure de conserver la garde de ses enfants. Ce syndrome comprend divers stratagèmes, diverses astuces pour empêcher le parent non gardien d'avoir accès à ses enfants en détruisant la figure parentale de ce dernier. Les enfants manifestent généralement un sentiment d'hostilité à l'égard de ce parent.

41. [1989] R.J.Q. 1361 (C.Q.).

42. Et de plus, l'article 16(10) de la Loi sur le divorce, L.R.C. 1985, $2^{\mathrm{e}}$ suppl., c. 3, stipule que les tribunaux doivent favoriser le plus de contacts possible de l'enfant avec chacun de ses parents. À cet égard, la décision dans Droit de la famille-1099, [1987] R.D.F. 236 (C.S.), respecte ce principe. 
intellectuellement apte de consentir à l'adoption ${ }^{43}$, ou encore lorsqu'on demande l'ouverture d'un régime de protection ${ }^{44}$.

En ce qui concerne la prise en charge d'un mineur dont la sécurité ou le développement est déclaré compromis ${ }^{45}$, il faudra évaluer les besoins de l'enfant afin de déterminer la ou les mesures les plus susceptibles de corriger la situation de compromission ${ }^{46}$. Les articles 86 à 88 L.P.J. reçoivent application et le tribunal devra alors obtenir une étude sur la situation sociale de l'enfant. On procédera à une analyse plus approfondie des personnes impliquées et, à cette fin, on a souvent recours à l'expertise pour identifier les mesures les plus susceptibles de favoriser le maintien de l'enfant dans son milieu ou, à tout le moins, lui procurer les meilleures conditions possible dans son intérêt ${ }^{47}$ et le respect de ses droits. Les travailleurs sociaux sont alors fréquemment appelés à intervenir auprès d'une famille afin que les comportements compromettants ne se reproduisent pas $^{48}$.

43. Droit de la famille-1779, [1993] R.J.Q. 1287 (C.Q.). Dans cette cause, malgré le fait que l'expert avait décrit l'intelligence de la demanderesse comme se situant à la limite inférieure de l'intelligence lente, le tribunal fut d'avis qu'elle n'était pas démunie au point d'être incapable de prendre des décisions ou qu'elle avait besoin d'une protection extraordinaire et refusa conséquemment de rétracter le consentement de la mère à l'adoption de son enfant. Dans une autre cause, il fut démontré au tribunal que la mère n'avait pas développé une autonomie suffisante pour assumer la garde de ses enfants, qu'elle demeurait dans un milieu inadéquat et était trop dépendante de ses parents. En outre, ses deux filles refusaient catégoriquement de la voir depuis presque trois ans. Le tribunal a déclaré les enfants judiciairement adoptables : Protection de la jeunesse-635, précité, note 14.

44. Trottier c. Trottier, [1992] R.D.F. 692 (C.S.); Lévesque c. Ouellet, [1990] R.J.Q. 2607 (C.S.). L'expert permettra au tribunal de déterminer le régime de protection qui conviendra le mieux à la personne jugée inapte.

45. Nous rappelons ici que l'enquête se déroule en deux étapes distinctes; la première visant à vérifier si le développement ou la sécurité de l'enfant est compromis, et la deuxième à déterminer les mesures à prendre pour corriger la situation.

46. Jacinthe MERCIER, «La procédure applicable en matière de protection de la jeunesse : régime contradictoire, inquisitoire ou mixte?», (1992) 22 R.D.U.S. 369, 390.

47. L'intérêt est défini de façon de plus en plus précise par les tribunaux. Voir à ce sujet Racine c. Woods, [1983] 2 R.C.S. 173; King c. Low, [1985] 1 R.C.S. 87; C. (G.) c. V.F. (T.), [1987] 2 R.C.S. 244.

48. MINISTÈRE DE LA JUSTICE DU CANADA, L'exploitation sexuelle des enfants dans la législation canadienne, Ottawa, Ministère des Approvisionnements et Services Canada, 1990, p. 101. 


\section{L'évaluation de la compétence parentale}

L'évaluation, qui vise à s'assurer que l'enfant sera confié au parent le plus apte à répondre à ses besoins, est souvent déterminante dans l'attribution de la garde des enfants ${ }^{49}$. Mentionnons également que l'analyse du choix du milieu parental approprié est une question de fait qui doit être déterminée en regard de chaque cas particulier ${ }^{50}$. Cependant, la compétence parentale n'est pas le seul facteur auquel réfère le tribunal. Ainsi, il pourra parfois accorder la garde conjointe, malgré le fait que la compétence parentale d'un parent présente certaines lacunes, au motif qu'il y va ainsi de l'intérêt de l'enfant ${ }^{51}$. Dans d'autres circonstances, lorsque l'évaluation de la compétence parentale n'est pas satisfaisante, le tribunal pourra tout simplement refuser d'accorder la garde de l'enfant à ce parent ${ }^{52}$.

Lors de l'évaluation des capacités parentales, l'expert devra tenir compte des liens affectifs développés entre le parent et l'enfant. À partir de ces constatations, il pourra faire des recommandations au tribunal concernant la garde. Lorsque le lien d'attachement de l'enfant avec l'un des parents est mis en péril, il faut prendre soin d'obtenir une évaluation impartiale des besoins réels de l'enfant avant d'attribuer le droit de garde ou de refuser un droit de visite à l'un des parents ${ }^{53}$. De ce qui précède, nous voyons qu'un parent peut être apte

49. Le professeur Claude Boisclair, dans Les droits et les besoins de l'enfant en matière de garde : réalité ou apparence?, Sherbrooke, Publication de la Revue de droit de l'Université de Sherbrooke, 1978, p. 158, écrit que l'expertise est une façon plus adéquate pour déterminer les besoins psychologiques de l'enfant et évaluer les aptitudes des parties à s'en occuper. Ainsi, dans Droit de la famille-1741, [1993] R.J.Q. 647 (C.A.), le tribunal a déclaré l'enfant admissible à l'adoption puisque l'attitude craintive et soumise de sa mère avait des répercussions négatives au plan de ses capacités parentales.

50. $\quad$ Protection de la jeunesse-309, J.E. 88-587 (T.J.).

51. Droit de la famille-1717, précité, note 40.

52. Droit de la famille-1242, précité, note 2, où le tribunal a refusé le changement de garde au motif que la mère ne permettait pas l'exercice libre et serein de la relation père-fils et que son équilibre psychologique était insatisfaisant.

53. Dans Droit de la famille-1385, précité, note 40, le père s'efforçait constamment de dénigrer la mère aux yeux de l'enfant lors de ses visites. Les rapports d'expertise psychologique concluaient que le père exerçait une influence négative sur l'enfant, mais soulignaient également la présence d'un lien affectif important entre l'enfant et le père. Le juge, se refusant alors de mettre entièrement fin aux droits de visite du père, les a cependant réduits, l'avertissant que ses droits pouvaient lui être totalement retirés s'il continuait à agir de la sorte. 
à assumer la garde d'un enfant, mais non pas du sien, si les liens affectifs développés avec lui ne sont pas suffisants ou s'ils sont inexistants ${ }^{54}$.

\section{L'évaluation des besoins psychologiques et affectifs de l'enfant}

Le tribunal devant statuer selon le meilleur intérêt de l'enfant, l'expert doit également, dans ses recommandations, tenir compte des besoins de celui- $\mathrm{ci}^{55}$. L'expert pourra aussi être amené à préciser s'il serait bénéfique pour l'enfant qu'une relation étroite s'établisse entre lui et son père ${ }^{56}$, ou encore à déterminer, au terme de la durée de l'interruption des droits d'accès d'un des parents, si l'enfant risquait de ne plus avoir de lien affectif avec lui ${ }^{57}$.

\section{B) L'évaluation de la capacité de l'enfant à rendre témoignage}

En vertu des articles 85.1 à 85.3 L.P.J., des psychologues peuvent, notamment, être appelés à évaluer si un enfant est intellectuellement apte à rendre témoignage devant le tribunal ou si le juge doit l'en dispenser au motif qu'il pourrait ainsi en subir un préjudice affectif grave. L'enfant pourra être jugé apte à témoigner, s'il est établi qu'il comprend l'importance de rapporter avec exactitude les faits dont il a eu connaissance ${ }^{58}$. L'expertise qui révélera que l'enfant est capable de comprendre la nature du serment fera présumer qu'il est apte à témoigner devant le tribunal.

La dispense de témoigner demandée pour un enfant apte à le faire ne peut être accordée que si on fait la preuve devant le tribunal d'un préjudice réel et suffisant ${ }^{59}$. Il semble qu'une évaluation psychologique sera souvent nécessaire

54. Voir à cet effet : Droit de la famille-1779, précité, note 43; Protection de la jeunesse-635, précité, note 14 .

55. Ce fut notamment le cas dans Protection de la jeunesse-406, 1989] R.D.F. 522 (C.Q.), où le témoin expert a déclaré que l'enfant souffrait de carence affective et qu'il était très agité. Il concluait à la nécessité d'un parent bien préparé pour s'occuper de lui et développer avec lui un lien affectif solide, stable et motivant.

56. $\quad$ Droit de la famille-1099, [1987] R.D.F. 236 (C.S.).

57. $\quad$ Droit de la famille-1715, J.E. 93-153 (C.A.).

58. L.P.J., art. 85.2.

59. Voir, à ce sujet, Protection de la jeunesse-434, [1990] R.J.Q. 1190 (C.Q.); Protection de la jeunesse - 471, [1991] R.J.Q. 564 (C.Q.); Protection de la jeunesse - 488, 1991] R.D.F. 244 (C.Q.). 
pour déterminer si l'enfant est apte à témoigner.

\section{CHAPITRE III : LES LIMITES DE L'EXPERTISE}

L'utilisation de connaissances relevant de sciences différentes pour régler un litige ne se fait pas sans certaines adaptations. L'expertise en est un bon exemple. Le droit et la science se côtoient tant bien que mal afin d'aboutir à la solution d'un litige. Cependant, chacune évolue dans son propre univers de concepts et chacune est limitée dans son cheminement par des facteurs que personne ne peut maîtriser.

Dans ce troisième chapitre, nous ferons état des limites de l'expertise imposées par le droit, d'une part, et par la science, d'autre part. Nous y discuterons également des principales différences conceptuelles qui les distinguent.

\section{Section 1: Les limites inhérentes au droit}

Le droit impose deux limites à l'expert. Tout d'abord, son expertise est restreinte au sujet relevant de sa compétence. Ensuite, il doit respecter le secret professionnel.

\section{A) L'expertise restreinte aux sujets relevant de la compétence de l'expert}

Lorsqu'un expert témoigne, il ne peut donner son opinion qu'en regard des sujets relevant directement de la compétence qu'il a acquise lors de ses études ou de son expérience professionnelle ${ }^{60}$. Il en résulte donc que le témoin expert n'est pas autorisé à se prononcer sur des questions de droit puisque seul le

60. La définition de «témoin expert» reflète cette idée : «Le témoin expert est celui qui possède une compétence spécialisée dans un secteur donné d'activité et qui a pour rôle d'éclairer le tribunal et de l'aider dans l'appréciation d'une preuve portant sur des questions scientifiques ou techniques» : J.-C. ROYER, op. cit., note 8, n 459, p. 169. «[...] Dans ces circonstances, on peut comprendre que les tribunaux cherchent à obtenir le plus d'aide possible de ceux qui peuvent être qualifiés d'experts. Ils peuvent donner un autre éclairage sur une preuve qui autrement serait de valeur négligeable [...]» : R. c. B. (G.), 1990] 2 R.C.S. 30, 38. 
tribunal est investi de la compétence nécessaire pour en disposer ${ }^{61}$. En conséquence, l'expert ne peut se prononcer sur la question finale que si elle concerne une question de fait ${ }^{62}$.

La distinction entre le droit et les faits a donc pour effet de limiter l'objet de l'expertise. Cette première limite comporte un autre volet qui consiste à réserver le qualificatif «d'expert» aux situations où l'expert se fonde véritablement sur ses connaissances particulières ${ }^{63}$. Autrement dit, la qualification «d'expert» dépend de la nature du témoignage que l'on rend et non pas de la qualité de la personne qui le rend. Cette distinction revêt une importance considérable, car en devenant un témoin ordinaire, l'expert sera soumis aux règles régissant le témoignage de celui-ci et perdra l'attribut le plus significatif du témoin expert, soit le pouvoir de rendre un témoignage d'opinion ${ }^{64}$.

\section{B) L'expertise restreinte par le secret professionnel}

La plupart des professionnels intervenant dans des litiges faisant appel à une certaine connaissance du comportement humain sont soumis à l'obligation de ne pas divulguer une information obtenue dans le cadre d'une relation professionnelle avec le bénéficiaire.

61. R. c. Béland, [1987] 2 R.C.S. 398, 415, où le tribunal a refusé d'entendre un expert au sujet des résultats obtenus par détecteur de mensonge pour le motif que dans «[...] notre système juridique [...] les juges et les jurys sont compétents pour déterminer la crédibilité et la fiabilité d'une preuve».

62. Ainsi, dans Protection de la jeunesse-323, [1988] R.J.Q. 1473, 1477 (T.J.), après avoir affirmé que les situations de compromission énoncées à l'article 38(g) de la Loi sur la protection de la jeunesse relèvent d'une appréciation factuelle et non juridique, le tribunal conclut : «Par conséquent, rien n'empêche le psychologue, en puisant dans son expérience professionnelle auprès des enfants, d'émettre son opinion sur le lien de causalité possible entre les perturbations diagnostiquées chez l'enfant et l'abus sexuel et de conclure que la cause de ce qu'il constate est ou n'est pas, selon lui, l'abus sexuel».

63. À ce sujet, voir : Daniel A. BELLEMARE, «L'homme de science devant les tribunaux», (1977) 37 R. du B. 465, 472.

64. Ibid., l'auteur nous donne l'exemple d'un médecin qui serait appelé à témoigner d'un accident dont il a été témoin. Bien qu'expert en médecine, son témoignage ne sera pas celui d'un expert. 


\section{Les informations obtenues par l'expert protégées par le secret professionnel}

Le droit au secret professionnel n'est pas un droit absolu. Avant de s'en prévaloir, une partie doit s'assurer de rencontrer les critères d'application et surtout, ne pas y avoir renoncé.

\section{a) Les critères d'application du secret professionnel}

L'article 9 de la Charte des droits et liberté de la personne a promu au rang de droit fondamental le secret professionnel. Cet article édicte que :

«Chacun a droit au respect au secret professionnel.

Toute personne tenue par la loi au secret professionnel et tout prêtre ou autre ministre du culte ne peuvent, même en justice, divulguer les renseignements confidentiels qui leur ont été révélés en raison de leur état ou profession, à moins qu'ils n'y soient autorisés par celui qui leur a fait ces confidences ou par une disposition expresse de la loi.

Le tribunal doit, d'office, assurer le respect du secret professionnel».

Les conditions d'application énoncées dans l'article 9 démontrent clairement qu'il ne s'agit pas d'un droit absolu ${ }^{65}$. Le critère essentiel, pour déterminer si la confidence faite à un professionnel est visée par l'article 9 de la Charte ou non, relève du type de relation qui existe entre ce dernier et le confident ${ }^{66}$.

65. Voir, entre autres, S.D. c. R.H., [1989] R.D.J. 390, 397 (C.S.); Nicole VALLIÈRES, «Le secret professionnel inscrit dans la Charte des droits et libertés de la personne du Québec», (1985) 26 C. de D. 1019, 1030, où il est énoncé que le secret professionnel ne s'applique que dans une relation professionnelle, qu'il ne protège que le client et que celui-ci peut y renoncer.

66. Léo DUCHARME, «Le secret médical et l'article 9 de la Charte des droits et libertés de la personne», (1984) 44 R. du B. 955, 959 : «[...] Si l'information recueillie doit servir à une autre fin que la prestation de services d'ordre personnel au confiant, l'obligation de confidentialité, même lorsqu'elle résulte de la loi, ne peut être considérée comme un secret professionnel». Ainsi, dans Protection de la jeunesse-435, [1990] R.D.F. 297, 300 (C.Q.), on a refusé de délier un professionnel de son secret pour protéger les déclarations qui lui ont été faites dans le cadre d'un suivi psychologique ordonné par le tribunal et ce, afin que cette ordonnance puisse être exécutée dans un climat d'entière confiance. 


\section{b) La renonciation volontaire au secret professionnel}

On peut renoncer de façon expresse ou implicite au secret professionnel ${ }^{67}$. La renonciation expresse ne pose pas de problème. Le bénéficiaire du droit au secret manifeste clairement son intention de ne pas s'en prévaloir. La renonciation implicite résulte de l'attitude de la personne. Le consentement à une évaluation psychosociale, ou le dépôt d'un rapport ${ }^{68}$, est interprété comme une renonciation tacite au secret professionnel ${ }^{69}$. L'obligation de se soumettre à une expertise entraîne le même résultat ${ }^{70}$ puisque, dans l'un et l'autre cas, les conditions pour invoquer le secret professionnel n'existent pas ${ }^{71}$.

La renonciation au secret professionnel affecte toutes les expertises subséquentes ayant un lien avec la première ${ }^{72}$. De plus, une fois délié du secret, l'expert peut venir témoigner en utilisant l'évaluation qu'il avait préparée pour une autre affaire ${ }^{73}$.

67. Jean-Pierre SENÉCAL, Droit de la famille québécois, Montréal, Publications CCH/FM ltée, 1985-1991, P54-220.

68. Ibid.

69. Voir Protection de la jeunesse-295, [1988] R.J.Q. 218, 220 (T.J.); Protection de la jeunesse435, précité, note 66, 299; Bernard GRATTON et Alexandra DRAGOMIR, «Aspects juridiques concernant la preuve de l'existence d'abus sexuel en matière de protection de la jeunesse, familiale ou criminelle», (1993) 23 R.D.U.S. 305, 329.

70. Droit de la famille-1922, J.E. 94-260 (C.Q.), p. 7, où le tribunal mentionne clairement : «Avant tout, il faut comprendre que l'évaluation psychologique ordonnée par le Tribunal au sens de l'article 87 L.P.J. n'est couverte par aucun secret professionnel».

71. Dans Protection de la jeunesse-483, [1991] R.D.F. 239, 241 (C.Q.), le tribunal refusa de protéger les informations obtenues par le professionnel alors que le père s'était soumis à l'ordre du tribunal lui enjoignant de se soumettre à une évaluation psychologique. Le tribunal motive sa décision ainsi : «Or, dans le cas de la fille, le père a reçu un ordre du Tribunal. Il n'était donc pas un «client» qui a requis ou choisi le professionnel. Il n'était pas un «patient» car il n'était d'aucune manière en relation d'aide par ce professionnel. Il n'était pas en consultation. Il n'avait aucune obligation contractuelle avec le professionnel. Il ne pouvait pas demander au professionnel ou lui ordonner de garder une certaine information secrète ni le professionnel pouvait-il lui garantir la confidentialité, car il devait faire rapport au Tribunal».

72. Protection de la jeunesse-432, précité, note 34,8 , où il est dit : «En se soumettant à la première expertise, le père a tacitement renoncé au secret professionnel en relation avec cette expertise ou avec toute contre-expertise qui en résulte.»

73. $\quad$ Voir à ce sujet : Protection de la jeunesse-483, précité, note 71. 


\section{Les informations non soumises au secret professionnel}

Toutes les informations que détient une personne, liée par le secret professionnel, ne sont pas nécessairement protégées ${ }^{74}$. Ainsi, les faits physiques constatés par un professionnel pendant une entrevue ne sont pas considérés comme des renseignements confidentiels protégés par la Charte. Il en est de même des opinions qu'il se fait à partir de ses propres constatations et qui ne sont pas fondées sur des confidences qui lui auraient été faites ${ }^{75}$.

\section{Section 2: Les limites inhérentes à la science}

La science n'est pas infaillible. Elle est limitée, d'une part, par la validité des tests eux-mêmes et, d'autre part, par des facteurs humains, telles les conceptions du professionnel et l'attitude de la personne soumise à ces tests.

\section{A) Les limites intrinsèques à la validité des tests}

Il arrive souvent qu'une expertise soit mise de côté parce que le tribunal n'est pas convaincu du principe fondamental ${ }^{76}$ sur lequel elle est fondée. Ainsi, la Chambre de la jeunesse, à la suite d'une entrevue faite par un psychologue avec des poupées sexuées, s'interroge sur la validité de tels tests :

«[... comment le juge peut-il déterminer la pertinence de ces éléments de preuve et en évaluer «les qualités de gravité, de précision et de concordance», s'il n'est pas éclairé quant à la validité du principe fondamental, quant à la fiabilité de la technique et enfin quant aux conditions d'application requises pour garantir des résultats probants?» ${ }^{77}$.

74. J-.P. SENÉCAL, op. cit., note 67, P54-220.

75. Dans l'affaire Protection de la jeunesse-197, [1985] T.J. 2025, 2031, il s'agissait de déterminer si le psychiatre était lié par le secret professionnel. Le tribunal a répondu négativement pour le motif que «[...] les informations apportées par le jeune lors de l'entrevue, que ses attitudes et ses comportements ne sont pas des faits «révélés» au sens de l'article 9 de la Charte».

76. La validité du principe fondamental est l'un des trois critères que l'opinion d'un scientifique doit rencontrer afin d'être crédible. Les deux autres critères sont les suivants : il faut établir la valeur probante de la technique appliquant le principe et, ensuite, il faut prouver l'application convenable de cette science au cas concret en litige : Pierre PATENAUDE, «De la recevabilité des preuves fondées sur des techniques non consacrées par la communauté scientifique», (1983) 43 R. du B. 51. 
La compétence de l'expert et la rigueur dont il fait preuve dans l'application des tests peuvent également influencer les résultats. En effet, lors de l'examen ou de l'évaluation, l'expert prend pour acquis certaines prémisses qui vont souvent influencer ses conclusions. Ainsi ${ }^{78}$, lors d'un examen gynécologique visant à établir s'il y avait eu abus sexuel ou non, le médecin découvre une encoche au niveau de l'hymen de la fillette. De ce fait, il a conclu catégoriquement que cette déchirure ne pouvait être causée que par un abus sexuel. Son témoignage fut contredit par un psychologue qui témoigna que cette déchirure pouvait être causée par des activités masturbatoires chez de jeunes enfants.

Le type d'évaluation privilégié par l'expert comporte en lui-même certaines limites. Ainsi, les résultats provenant d'une évaluation microscopique ${ }^{79}$ nous permettent de connaître le fonctionnement mental d'un individu, mais ils ne permettent pas d'évaluer les interactions avec les autres membres de sa famille ${ }^{80}$, et tout ce qu'il pourrait en dire ne serait que pure théorie ${ }^{81}$. D'un autre côté, l'évaluation macroscopique est de loin l'approche la plus appropriée en matière familiale. Elle permet à l'expert de prendre connaissance des relations qu'un individu entretient avec son milieu et d'évaluer chacun des individus tout en ayant une approche plus globale ${ }^{82}$.

77. $\quad$ Protection de la jeunesse-329, [1988] R.J.Q. 1739, 1748 (T.J.).

78. $\quad$ Protection de la jeunesse-634, J.E. 93-1296 (C.Q.), p. 15.

79. Pour une étude plus approfondie de ce type d'évaluation, voir : Luc MORIN, «Les techniques d'évaluation de l'intervenant psycho-social devant le Tribunal de la jeunesse et la Cour supérieure», (1981) 11 R.D.U.S. 591, 593-596.

80. Il en est ainsi, par exemple, lorsqu'il s'agit de déterminer si une personne est apte à consentir à des soins médicaux. À cet effet, voir : Hôpital Charles-Lemoyne c. Forcier, [1992] R.D.F. 257 (C.S.); Douglas Hospital Center c. T. (C.), précité, note 39; Hôpital St-CharlesBorromée c. G. (G.), précité, note 39.

81. L. MORIN, loc. cit., note 79, 594-595, où l'auteur illustre ce propos.

82. L'affaire $X$. c. Z., précitée, note 3,509, est un excellent exemple de ce type d'évaluation. Le tribunal mentionne que l'expertise qui lui est présentée est très bien conçue puisqu'elle analyse «la situation familiale actuelle des parties, l'évolution de leur situation conjugale, le profil de la requérante, de l'intimé et des enfants [...]». 


\section{B) Les limites concernant les variables incontrôlables}

Nous qualifions ces variables d'incontrôlables parce qu'elles sont indissociables de la nature humaine. Elles font appel aux convictions personnelles de chacun.

\section{Les conceptions du professionnel}

Dans l'appréciation d'une expertise, il est indispensable de connaître les croyances auxquelles le professionnel adhère ${ }^{83}$. Les préjugés du professionnel peuvent être inhérents à sa profession. À ce sujet, $\mathrm{M}^{\mathrm{e}}$ Boisclair écrit :

«Les sciences psychosociales ne sont pas des sciences exactes et les spécialistes pratiquant les expertises peuvent avoir des conceptions différentes. Aussi, il nous paraît utile, voire même essentiel, que l'avocat puisse contre-interroger l'expert sur sa façon de procéder, sur ses recommandations et les faits sur lesquels il s'appuie pour justifier son rapport» ${ }^{84}$.

Dans les domaines où l'expertise relève d'une appréciation subjective, les préjugés de l'expert risquent de venir teinter ses conclusions. Ce phénomène est courant en matière d'abus sexuels. Ainsi, le spécialiste qui entreprend son investigation avec la croyance voulant que les enfants ne mentent jamais lorsqu'ils révèlent qu'ils sont victimes d'abus sexuels ${ }^{85}$, s'enferme dans des préjugés qui l'empêchent d'étudier toutes les possibilités susceptibles d'expliquer les déclarations de l'enfant à ce propos. Il est victime de l'effet appelé «Rosenthal», qui est le phénomène par lequel l'observateur qui attend ou anticipe un

83. Un exemple suffira pour vous en convaincre. Il s'agit d'une affaire où il faut déterminer si l'habitude de la mère consistant à embrasser sa fille sur les parties génitales constitue un abus sexuel. Les experts sont divisés, certains y voient un abus alors que d'autres n'y voient que «des gestes d'intimité affective entre une mère et son enfant», Protection de la jeunesse-380, [1989] R.J.Q. 1037, 1044 (C.Q.).

84. C. BOISCLAIR, op. cit., note 49, p. 164.

85. Hubert VAN GIJSEGHEM, «L'enfant témoin : facteurs cognitifs», dans L'enfant abusé : psychologie et droit (1992), Cowansville, Éditions Yvon Blais inc., 1992, p. 27, à la page 36. L'auteur explique que «la connaissance qu'a l'enfant de la sexualité, et les fantaisies qu'il entretient autour de ce thème, sont immensément plus diversifiées et élaborées que l'adulte le plus libéral pourrait bien imaginer.» 
résultat a de bonnes chances de le trouver ${ }^{86}$.

\section{L'attitude de la personne soumise à l'expertise}

L'expertise portant sur le comportement humain oblige à prendre en considération les aléas psychologiques, et plus particulièrement lorsqu'il s'agit de jeunes enfants. Deux facteurs affectent les propos d'un enfant. Il y a d'abord sa mémoire qui n'a pas encore atteint sa pleine maturité, ce qui le rend vulnérable à la suggestion ${ }^{87}$. Ensuite, l'enfant très influencé par l'adulte qui le questionne, sera tenté de se conformer à ses attentes en répondant à ses questions $^{88}$. L'expert ou le juriste qui aura à évaluer ou à questionner un enfant doit éviter d'influencer l'enfant de quelque façon que ce soit s'il désire obtenir un récit véridique ${ }^{89}$. De plus, le déroulement de l'expertise contient en lui-même du matériel suggestif telles des images, des poupées sexuées ou des questions suggestives. Ce matériel vient colorer son récit et il a souvent pour effet de le modifier de telle sorte qu'il lui sera impossible de recouvrer l'information d'origine $^{90}$.

Dans Protection de la jeunesse-659, [1994] R.J.Q. 236, 246 (C.Q.), la cour, traitant de la difficulté du rôle du travailleur social en tant qu'expert, reprend les propos du psychologue Hubert Van Gijseghem et énonce ce qui suit : «Le travailleur social a un mandat : il doit protéger l'enfant potentiellement en danger. Il entreprend donc son travail avec un postulat opposé à celui du représentant du système judiciaire : quand il y a un signalement, il faut croire au départ qu'il y a abus, car il ne peut se permettre de ne pas agir dans un vrai cas d'abus».

87. Luc MORIN et Claude BOISCLAIR, «La preuve d'abus sexuel : allégations, déclarations et l'évaluation d'expert», (1992) 23 R.D.U.S. 27, 36.

88. H. VAN GIJSEGHEM, op. cit., note 85, p. 27, à la page 34.

89. Pour des exemples d'interrogatoires suggestifs, voir : Protection de la jeunesse-233, [1987] R.J.Q. 2701, 2705 (T.J.), où le psychologue questionne l'enfant lors d'une entrevue; Protection de la jeunesse-468, [1991] R.D.F. 94, 95 (C.Q.), où c'est la gardienne qui a interrogé l'enfant; Protection de la jeunesse-659, précité, note 86 , où on relate l'interrogatoire de l'enfant devant le tribunal.

90. H. VAN GIJSEGHEM, op. cit., note 85, p. 27, à la page 34. L'arrêt Protection de la jeunesse-578, J.E. 92-1710 (C.Q.), illustre ce problème. Dans cette affaire, on soupçonnait le père d'avoir abusé de ses deux fillettes. Au début de l'enquête, celles-ci nient catégoriquement les abus, mais les intervenants continuent de poser des questions. Plus tard, l'enquêteur remet à l'une d'elles un livre sur «le secret». Quelques jours plus tard, elle avoue avoir été victime d'abus sexuels. Sa soeur, de son côté, dénoncera l'abus sexuel après avoir suivi quelques séances de thérapie pour enfants abusés sexuellement. Le tribunal se questionne alors sur l'opportunité de faire suivre de telles thérapies alors que l'abus n'est pas encore prouvé. Cet arrêt illustre bien à quel point les enfants peuvent parfois être soumis à 
Plusieurs autres facteurs peuvent intervenir dans le processus de l'expertise. En effet, autant l'adulte que l'enfant seront influencés par le délai écoulé depuis la survenance des faits. Leur âge, leurs expériences respectives et leur réceptivité pourront également influer sur les conclusions de l'expertise. Ainsi, l'enfant placé dans un contexte de divorce pourra être confronté à un conflit de loyauté qui le poussera à agir dans l'intérêt d'un seul de ses parents ${ }^{91}$. L'adulte, lui, pourra souffrir du syndrome de l'aliénation parentale qui pourra modifier sa perception des événements ${ }^{92}$.

\section{Section 3 : La conciliation du droit et de la science}

La science et le droit ont tous les deux comme objectif la recherche de la vérité. Cependant les deux procèdent différemment. Il est donc important de connaître ces différences pour intégrer correctement les données scientifiques au domaine juridique.

Le scientifique, dans sa quête de la vérité, utilise comme référence la règle générale alors que le juriste doit la rechercher dans le cas d'espèce ${ }^{93}$. Pour le premier, la vérité repose sur une base objective et vérifiable, alors que pour le second, elle est fondée sur des éléments fugaces ${ }^{94}$. De plus, l'appréciation de la probabilité est la difficulté la plus considérable survenue depuis l'acceptation de l'expert dans les cours de justice ${ }^{95}$. Il faudra donc sensibiliser l'expert au fait que le degré de certitude requis en droit est moins exigeant que celui requis en science afin que celui-ci en tienne compte dans ses interventions ${ }^{96}$.

\footnotetext{
la suggestion.

91. Protection de la jeunesse-622, J.E. 93-968 (C.Q.), où l'on relate que l'enfant en conflit de loyauté est plus sensible à la suggestion.

92. $\quad$ Pour un exemple, voir Droit de la famille-1717, précité, note 40.

93. Charles D. GONTHIER, «Le témoignage d'experts : à la frontière de la science et du droit», (1993) 53 R. du B. 187, 194; voir également : Daniel JUTRAS, «Expertise scientifique et causalité», dans Congrès annuel du Barreau du Québec (1992), p. 897, à la page 903.

94. C.D. GONTHIER, ibid.

95. Katherine LIPPEL, «L'incertitude des probabilités en droit et en médecine», (1992) 22 R.D.U.S. 445; voir également : D. JUTRAS, op. cit., note 93, pp. 904 et suivantes.

96. Ainsi, la Cour suprême fait une mise en garde importante en ce qui a trait à la distinction entre probabilité juridique et probabilité scientifique dans l'affaire Snell c. Farrell, 1990] 2 R.C.S. 311, 330, «[...] il n'est pas essentiel que les experts médicaux donnent un avis ferme à l'appui de la théorie de la causalité du demandeur. Les experts médicaux déterminent habituellement l'existence de causalité en des termes de certitude, alors qu'une norme
} 


\section{DEUXIÈME PARTIE : L'INFLUENCE DE L'EXPERTISE SUR L'ADJUDICATION D'UN LITIGE EN MATIÈRE FAMILIALE}

L'expertise doit au préalable être reçue par les tribunaux pour lui reconnaître des effets. En effet, il faut distinguer la recevabilité de la preuve par expert de son admissibilité. Les auteurs J.-L. Baudouin et Y. Renaud écrivent à ce sujet :

«Il y a lieu de distinguer l'admissibilité d'une preuve par témoins de la réception d'une telle preuve. Admettre la preuve testimoniale est juger qu'elle est valablement introduite, tout en lui attribuant plus ou moins force probante. Recevoir la preuve testimoniale, est accepter de l'entendre sans nécessairement décider immédiatement de sa légalité» ${ }^{97}$.

Il faut comprendre ici qu'on parle de la légalité au niveau de la recevabilité de la preuve, c'est-à-dire de l'introduction valable d'une preuve. Il n'est pas question de «légalité» au niveau de l'admissibilité puisque le juge n'apprécie alors pas la «légalité» de la preuve, mais bien sa valeur probante.

Ces deux étapes sont clairement définies, tant au niveau de la doctrine que la jurisprudence, mais ces dernières emploient parfois les termes «recevabilité» et «admissibilité» comme synonymes ${ }^{98}$ pour déterminer si la preuve est

inférieure est exigée par le droit». Dans l'affaire Droit de la famille-1618, précitée, note 39, 6 , le tribunal se questionne en ces termes quant à la pertinence d'une question posée à un psychiatre faisant appel à la «certitude médicale» : «It is difficult to see how a question to a psychiatrist as to the «certitude médicale» of the evidence given by another psychiatrist and a psychologist can be relevant, particularly when the expert had never seen the respondent. Of what relevance is «certitude médicale» when that is not the standard of proof required in a civil suit?»

97. Jean-Louis BAUDOUIN et Yvon RENAUD, Code civil du Québec annoté, vol. 4, Montréal, Wilson et Lafleur ltée, 1990, p. 124, sous l'ancien article 589 C.c.Q. Déjà en 1947, dans G. c. P., [1947] B.R. 99, 101, le juge Pratte énonçait en ces termes la distinction entre la recevabilité et l'admissibilité d'une expertise en déclarant : «La réception d'une preuve n'implique pas nécessairement son admissibilité. Une preuve à laquelle il y a eu objection n'est définitivement admise que lorsque le juge en tient compte pour en tirer des conséquences; jusque-là, elle n'est que reçue».

98. Voir, par exemple, Protection de la jeunesse - 605, précité, note 38 , où on se demandait si le témoignage d'un témoin expert portant sur le comportement général et les caractéristiques psychologiques des enfants victimes d'abus sexuels était «admissible» en preuve. Le tribunal en est venu à la conclusion que le témoignage de l'expert était un témoignage pertinent et 
recevable et non pas si elle est probante. Si l'admissibilité est synonyme de force probante, elle ne peut être synonyme de recevabilité, d'où l'intérêt de faire le point. Nous croyons utile de conserver la distinction énoncée par les auteurs J.L. Baudouin et Y. Renaud.

Pour les fins de notre exposé, la recevabilité signifie accepter d'entendre l'expert ou accepter le dépôt de son expertise, tandis que l'admissibilité c'est donner effet au témoignage ou au rapport en déterminant le degré de force probante de cette preuve. Donc,

«[... de façon générale, le problème de l'expertise n'existe pas vraiment au niveau de sa recevabilité, mais plutôt, comme l'indique la Cour suprême, au niveau de son admissibilité qui soulève la question de la valeur probante à accorder à l'opinion de l'expert» ${ }^{99}$.

\section{CHAPITRE I : LA RECEVABILITÉ DE LA PREUVE PAR EXPERT}

Le tribunal n'acceptera d'entendre une preuve par expert qu'à certaines conditions et ce n'est qu'une fois celles-ci respectées que l'expert pourra témoigner sur les faits du litige et qu'il pourra éventuellement influencer le sort du litige. Après avoir précisé ce qu'il faut entendre par «recevabilité», nous aborderons les conditions de recevabilité et de forme de la preuve par expert.

\section{Section 1: La notion de recevabilité de la preuve par expert}

La Cour suprême a précisé le rôle du témoin expert en ces termes :

«Quant aux questions qui exigent des connaissances particulières, un expert dans le domaine peut tirer des conclusions et exprimer son avis. Le rôle d'un expert est précisément de fournir au juge et au jury une conclusion toute faite

«recevable». Voir également Droit de la famille - 1910, J.E. 94-175 (C.S.); MIUF-12, [1988] R.D.J. 455 (C.S.); Protection de la jeunesse-323, [1988] R.J.Q. 1473 (T.J.); Protection de la jeunesse-539, [1992] R.J.Q. 1144 (C.Q.); J.-C. ROYER, op. cit., note 8, ${ }^{\circ}$ 460, p. 169.

99. L. MORIN et C. BOISCLAIR, loc. cit., note 87, 63; de même, dans Mathieu c. Beauceville (Corp. de la Ville de), J.E. 93-594 (C.S.), on affirme que le témoignage de l'expert est soumis à l'appréciation du juge et c'est alors sa force probante qui est en cause, et non sa recevabilité. 
que ces derniers, en raison de la technicité des faits, sont incapables de formuler» ${ }^{100}$.

Les tribunaux ont donc établi clairement qu'une preuve d'expert n'est recevable que lorsque la preuve profane ne suffit pas, c'est-à-dire quand les faits sont trop complexes pour être résolus par une personne ayant des connaissances ordinaires $^{101}$. Le tribunal doit donc, avant de déclarer recevable une preuve d'expert, constater la nécessité d'un éclairage particulier sur la question à trancher ${ }^{102}$.

\section{Section 2 : Les critères de recevabilité de l'expertise}

La recevabilité d'une expertise est subordonnée à deux conditions : d'une part, elle doit être nécessaire à la compréhension des faits du litige et, d'autre part, elle doit être pertinente à celui-ci.

\section{A) La nécessité de l'expertise}

Le critère de la nécessité avait été clairement énoncé par l'honorable juge Dickson en $1982^{103}$, lorsqu'il précisa que l'opinion de l'expert n'était pas nécessaire lorsque le juge peut tirer ses propres conclusions à partir des faits

100. R. c. Abbey, [1982] 2 R.C.S. 24, 42. Voir au même effet Léo DUCHARME, «La prohibition du ouï-dire et les déclarations des enfants en bas âge au sujet des abus sexuels dont ils auraient été victimes», (1987) 18 R.G.D. 563, 592; R. c. Béland, précité, note 61. Mentionnons que les principes énoncés en matière pénale s'appliquent en droit civil compte tenu de l'origine de nos règles d'administration de la preuve : J.-C. ROYER, op. cit., note 8 , $\mathrm{n}^{\circ} 469$, p. 172.

101. RAPPORT DU GROUPE DE TRAVAIL SUR L'ÉVALUATION DE LA LOI SUR LA PROTECTION DE LA JEUNESSE, La protection de la jeunesse, plus qu'une loi, Québec, Ministère de la Santé et des Services sociaux et Ministère de la Justice, 1992, p. 121.

102. R. c. Nielsen and Stolar, (1985) 16 C.C.C. (3d) 39 (C.A. Man.). Voir aussi Michael Newman HOWARD, Peter CRANE et Daniel A. HOCHBERG, Phipson on evidence, $14^{\mathrm{e}}$ éd., London, Sweet \& Maxwell, 1990, n³2-07, p. 804, qui écrivent qu'en Grande-Bretagne le recours à l'avis d'un expert doit être limité aux sujets sur lesquels la compétence pour se former une opinion ne peut être acquise que par des études spéciales ou par l'expérience.

103. R. c. Abbey, précité, note 100, 42. Voir également, dans le même sens, Protection de la jeunesse-329, précité, note 77, 1747; R. c. Nielson and Stolar, précité, note 102; L. MORIN et C. BOISCLAIR, loc. cit., note 87, 62. 
établis par la preuve ${ }^{104}$. Le témoignage de l'expert est donc irrecevable s'il n'éclaire pas le tribunal.

Il revient au juge de décider quand des explications de personnes objectives et spécialisées, connaissant bien les règles de leur art, lui sont nécessaires pour comprendre les faits en litige. Signalons qu'une expertise peut être qualifiée d'«utile» lorsque le point de vue d'un spécialiste est intéressant, mais elle n'est pas indispensable au juge en mesure d'apprécier lui-même les faits. Il s'agit d'une expertise non nécessaire quoiqu'elle ait pu être utile ${ }^{105}$. La restriction quant au témoignage des experts se comprend aisément : le juge étant le seul à avoir juridiction pour décider du litige, il n'a pas besoin d'entendre un spécialiste s'il est à même d'apprécier seul la preuve ${ }^{106}$.

104. Récemment, le Rapport du groupe de travail sur l'évaluation de la Loi sur la protection de la jeunesse, op. cit., note 101, p. 131, reprenait à son compte ce critère en ces termes : «Si par ailleurs, les parties et leur procureur, conscients de la gravité du processus et de ses séquelles, agissent en matière d'expertise avec respect, réserve et sobriété, on pourra espérer un usage plus restrictif et plus proche du critère de nécessité qui, de toute façon, régit la preuve d'expert. Rappelons qu'en effet, la jurisprudence a clairement établi qu'une preuve d'expert n'est admissible que là où la preuve profane ne suffit pas, c'est-à-dire quand les faits sont trop complexes pour être compris par l'homme ordinaire ou moyen».

105. J.-C. ROYER, op. cit., note 8, considère toutefois, au $n^{\circ} 460$ de la page 169 , l'utilité de l'expertise et non sa nécessité comme l'une des conditions de recevabilité du témoignage de l'expert.

106. Dans la décision R. c. Scopelliti, (1982) 63 C.C.C. (2d) 481 (C.A. Ont.), 499, la Cour d'appel de l'Ontario déclare : «[...] [That] evidence with respect to the other matters previously outlined did not fall within the proper sphere of expert testimony because it was superfluous and related to matters falling within the common stock of knowledge with respect to which the jurors were competent to form their own judgment». Déjà en 1931, dans Village of Kelliher c. Smith, [1931] R.C.S. 672, 684, la plus haute instance judiciaire du pays avait clairement énoncé que pour faire l'objet d'une expertise, le sujet doit être tel que les gens ordinaires sont incapables de formuler un jugement correct s'ils ne sont pas assistés par une personne qualifiée. 


\section{B) La pertinence de l'expertise}

Il existe en matière de preuve un principe général selon lequel une preuve doit être pertinente pour être recevable ${ }^{107}$. L'expertise, en tant que moyen de preuve, ne fait pas exception à cette règle. Le professeur Ducharme a défini ainsi la pertinence :

«Pour déterminer la pertinence d'un élément de preuve, il faut l'apprécier au regard de l'objet même du litige. De façon générale, on peut dire que tout élément de preuve qui tend à établir l'existence ou la non-existence du droit réclamé est pertinent.

[...] De façon générale, il est possible d'affirmer qu'il suffit qu'un fait présente un lien logique avec l'objet du litige et les questions incidentes qu'il soulève pour satisfaire au critère de la pertinence» ${ }^{108}$.

On peut en déduire que la «pertinence» concerne la qualité ainsi que la proximité du lien entre le fait mis en preuve et le fait à prouver ${ }^{109}$. Le tribunal doit ainsi se demander s'il existe un lien entre le sujet de l'expertise et la

107. La règle, qui sous le C.c.B.-C. était non écrite, est maintenant codifiée à l'article 2857 C.c.Q. en ces termes : «La preuve de tout fait pertinent au litige est recevable». Nous discuterons plus loin de deux exceptions importantes à ce principe, soit la preuve par ouï-dire et le témoignage d'opinion : Infra., Partie II, Chapitre II, pp. 49 et suivantes.

108. Léo DUCHARME, Précis de la preuve, 4 éd., Montréal, Wilson et Lafleur ltée, 1993, ${ }^{\circ}$ 739, p. 244. À cet égard, l'arrêt R. c. Cloutier, [1979] 2 R.C.S. 709, 731 et 733, souvent cité, a établi que : «Pour qu'un fait soit pertinent à un autre, il faut qu'il existe entre les deux un lien ou une connexité qui permette d'inférer l'existence de l'un à raison de l'existence de l'autre. Un fait n'est pas pertinent à un autre s'il n'a pas par rapport à celui-ci une valeur probante véritable. [...] La pertinence d'un fait que l'on veut mettre en preuve doit évidemment s'apprécier en regard de la nature du litige et des diverses questions qui y sont en jeu». Commentant cette décision de la Cour suprême, le juge, dans Protection de la jeunesse, C.Q. Laval, 540-41-000183-888, p. 16 du jugement, s'exprimait ainsi : «Toute déduction de fait mis en preuve en l'absence de ce lien ou connexité ne serait que pure spéculation, conjecture ou soupçon».

109. C.D. GONTHIER, loc. cit., note 93. L'honorable juge Godin, dans Protection de la jeunesse-539, précité, note 98, a effectué une analyse applicable à toutes expertises ou preuves scientifiques que l'on cherche à introduire en preuve lors d'une audition. Il s'agissait en l'espèce d'une preuve par pléthysmographe et le juge conclut que la recevabilité, mais surtout la valeur probante, dépendait de la possibilité d'inférer en toute logique une connexité entre les données recueillies et l'abus sexuel. La pléthysmographie est un test qui consiste à fixer un anneau autour du pénis afin de capter les variations de son volume et ainsi voir s'il réagit à certains stimuli. 
question à trancher ${ }^{110}$. Notons que si le principe de la pertinence constitue un critère important pour décider de la recevabilité d'une preuve par expert, il l'est également pour déterminer le poids du témoignage de l'expert, une fois reçu en preuve $^{111}$. En dernier lieu, mentionnons que, dans le cadre d'une expertise psychosociale en matière familiale, on doit, d'après les Règles de pratique ${ }^{112}$, démontrer la pertinence et l'opportunité de l'expertise.

\section{Section 3 : Les conditions de forme de l'expertise}

Les formalités préalables à la production d'une preuve par expert diffèrent selon que l'on se retrouve en matière familiale ou dans le contexte particulier de la protection de la jeunesse. Examinons les conditions de forme relatives à chacune de ces situations.

\section{A) Les règles générales en matière familiale}

Les expertises produites lors d'un litige en matière familiale sont généralement soumises à trois règles. Aucun expert ne peut être entendu sans que son rapport n'ait été préalablement déposé. De plus, le tribunal ne peut ordonner une expertise que si les parties y ont préalablement consenti et que son opportunité est démontrée.

110. On avait décidé, dans Protection de la jeunesse-438, J.E. 90-765 (C.Q.), que le témoignage de l'expert concernant le profil d'un parent abuseur ou d'un enfant abusé n'établissait pas un lien de connexité suffisant et ne tendait pas à établir s'il y avait eu, en l'espèce, une situation d'abus. Le témoignage du psychologue avait donc été déclaré irrecevable.

111. C.D. GONTHIER, loc. cit., note 93, 187.

112. R.p.C.s. mat. fam., art. 23.3. 


\section{Le dépôt du rapport de l'expert}

Le dépôt du rapport est l'une des formalités à respecter ${ }^{113}$ avant de permettre à un expert de témoigner ${ }^{114}$, tel que le prévoit l'article 402.1 C.p.c. ${ }^{115}$. À cette règle correspond deux exceptions. Premièrement, le tribunal peut, à sa discrétion et suivant les justifications de la partie en défaut, autoriser l'expert à témoigner sans l'accomplissement de cette formalité ${ }^{116}$, si l'inobservation de l'article 402.1 C.p.c. ne cause pas préjudice à l'autre partie ${ }^{117}$. Le juge peut également ajourner l'audition pour lui permettre de se conformer à la règle.

La deuxième exception a trait au dépôt du rapport d'un expert après la production du certificat d'état de cause. La permission du tribunal est également nécessaire et elle «ne sera accordée que dans des cas exceptionnels» ${ }^{118}$.

113. Les deux parties doivent savoir avant le jour du procès ce qui les attend (C.p.c., art. 397 à 403). Il s'agit d'une manifestation claire du législateur de son intention d'éviter que l'une ou l'autre des parties soit prise par surprise, de favoriser la divulgation de la preuve et un règlement, le cas échéant, et d'assurer que le dossier qui vient pour audition est complet.

114. L'amendement à l'article 402.1 C.p.c. en 1985 constitue un changement radical comparativement à l'ancien texte. Celui-ci n'empêchait pas un expert de témoigner verbalement devant le tribunal sur le contenu de son rapport, et ce même s'il y avait défaut d'observer la condition imposée par l'ancien article 402.1 C.p.c. Maintenant, nul expert n'est autorisé à témoigner à moins d'avoir produit son rapport écrit dans les délais prévus par les Règles de pratique.

115. Tel que modifié par la Loi modifiant le Code de procédure civile et d'autres dispositions législatives, L.Q. 1984, c. 26, a. 18.

116. Watson c. Sutton, [1990] R.D.J. 175 (C.A.).

117. J.-C. ROYER, op. cit., note 8, no 473, p. 174.

118. R.p.C.s. mat. fam., art. 17. De plus, le professeur J.-C. Royer, op. cit., note 8, $\mathrm{n}^{\circ}$ 473, p. 173, affirme que certaines décisions rendues, en vertu de l'article 294.1 C.p.c., s'appliquent mutatis mutandis à l'article 402.1 C.p.c. et que, conséquemment, le défaut de comparaître ou de plaider ne dispense pas le demandeur de se conformer à cette règle : Bergeron $\mathrm{c}$. Couture, [1972] R.P. 422; Stauber c. Coyle, [1971] R.P. 191; Léo DUCHARME, «Le régime spécial des rapports produits en vertu de l'article 294.1 C.P.», (1980) 40 R. du B. 822. Le simple dépôt du rapport d'un expert est insuffisant si un avis et une copie ne sont pas signifiés à l'adversaire : Beare c. Racine, [1969] R.P. 188; L. DUCHARME, op. cit., note 16, n ${ }^{\circ} 417$, p. 138. 


\section{La preuve de l'opportunité}

Lorsque le litige implique des enfants mineurs ${ }^{119}$, le juge rendra une ordonnance d'expertise psychosociale ${ }^{120}$ s'il est établi à sa satisfaction qu'il y a un début de preuve lui permettant de décider de son opportunité. En effet, l'article 23.3 des Règles de pratique de la Cour supérieure en matière familiale en fait une condition impérative :

«Toute ordonnance d'expertise psychosociale est précédée d'une preuve permettant au tribunal d'en décider l'opportunité. Le juge demeure saisi du dossier à moins que pour des raisons d'ordre administratif, il ne s'en désaisisse expressément».

\section{L'accord des parties}

Dans les cas d'une expertise en vertu des Règles de pratique de la Cour supérieure en matière familiale, par exemple dans les cas de garde et de droits d'accès, le juge doit préalablement obtenir le consentement des parties ${ }^{121}$ et celui de l'enfant s'il est âgé de quatorze ans et plus, avant de pouvoir ordonner qu'une expertise psychosociale soit faite. Le rapport terminé, le Service d'expertise psychosociale le transmet au juge saisi de la cause qui le transmet à son tour aux procureurs $^{122}$. Le rapport de l'expert fait ensuite partie de la preuve et l'expert peut être appelé à témoigner ${ }^{123}$.

\section{B) Les règles particulières à la Loi sur la protection de la jeunesse}

Les expertises faites sous l'empire de la Loi sur la protection de la jeunesse ne sont pas soumises à l'article 402.1 C.p.c. et aux articles 414 et suivants du

119. R.p.C.s. mat. fam., art. 23.1.

120. Suivant les articles 23 à 24 R.p.C.s. mat. fam. On aura alors recours au service d'expertise psychosociale de la Cour supérieure.

121. R.p.C.s. mat. fam., art. 23.2. Les parties doivent signer le consentement à l'expertise et le juge émettra par la suite son ordonnance.

122. R.p.C.s. mat. fam., art. 24.

123. R.p.C.s. mat. fam., art. 23.6. 
C.p.c. ${ }^{124}$. Notons, toutefois, qu'une certaine jurisprudence interprète de façon large l'article 85 L.P.J. et estime que les autres dispositions du Code de procédure s'appliquent et qu'il n'aurait pas pour effet d'exclure les autres dispositions compatibles ${ }^{125}$.

\section{Le principe : la situation de compromission}

En matière de protection, l'enquête se déroule en deux temps. La première étape consiste à déterminer s'il existe une situation de compromission, tandis que la seconde étape concerne l'enquête sur les mesures à prendre à l'égard de l'enfant $^{126}$.

Lors de l'enquête sur la situation de compromission, la lecture de l'article 86 L.P.J. nous amène à conclure que le tribunal n'aurait pas le pouvoir d'ordonner, de sa propre initiative, une expertise ou une évaluation psychosociale ${ }^{127}$. Il ne pourra le faire qu'au niveau de la deuxième étape, celle qui vise les mesures à prendre. Toutefois, en vertu du courant jurisprudentiel voulant que l'article 85 L.P.J. soit interprété largement, il semble que le tribunal pourrait demander, à cette étape, une expertise en se fondant sur le Code de procédure.

124. En effet, $\mathrm{M}^{\mathrm{e}} \mathrm{J}$.-F. Boulais affirme, dans Loi sur la protection de la jeunesse, texte annoté, op. cit., note 40, p. 318, que les règles édictées par ces dispositions, dont le fait que le témoin expert ne pourrait être entendu que si son rapport a été préalablement signifié aux parties, ne trouveraient pas d'application en matière de protection de la jeunesse parce qu'elles n'apparaissent pas dans l'énumération de l'article 85 L.P.J. et qu'elles sont jugées incompatibles avec les autres dispositions de la loi. Voir également: Re Centre des Services sociaux du Québec, [1980] R.P. 421 (C.S.); Protection de la jeunesse-157, J.E. 85-165 (T.J.).

125. Voir, entre autres, Protection de la jeunesse-211, [1986] R.J.Q. 1377 (T.J.), où le tribunal, prétextant que l'article 85 L.P.J. réfère à l'article 20 C.p.c., estime qu'il peut se référer aux autres articles du C.p.c. non prévus par l'article 85 L.P.J. Voir dans le même sens Protection de la jeunesse-363, [1989] R.J.Q. 206 (C.Q.).

126. Ainsi, dans Protection de la jeunesse-139, [1984] T.J. 2067, on ordonna l'hébergement en centre d'accueil d'un enfant jusqu'à la date de l'enquête au fond de la demande principale, mais le tribunal se déclara sans juridiction à ce stade des procédures pour ordonner une évaluation psychologique ou encore des recommandations en ce sens puisque la sécurité et le développement de l'enfant n'avaient pas encore été déclarés compromis.

127. Article 86 L.P.J. : «Avant de rendre une décision sur les mesures applicables, le tribunal doit demander au directeur de faire une étude sur la situation sociale de l'enfant. Le directeur peut, à sa discrétion, ou doit, si le tribunal le requiert, y joindre une évaluation psychologique ou médicale de l'enfant et des membres de sa famille ou toute autre expertise qui peut être utile $[\ldots]$... 


\section{L'exception : le consentement des parties}

Au niveau de l'enquête sur la compromission, les parties au litige sont les seules à pouvoir recourir aux services d'un expert ${ }^{128}$. Il est également possible de recourir à la contre-expertise dans la mesure où elle est destinée à vérifier les conclusions d'une première expertise. En effet, les dispositions de la Loi sur la protection de la jeunesse et la jurisprudence affirment qu'il est possible de demander une contre-expertise tant au niveau de l'enquête sur la compromission qu'à l'enquête sur les mesures de protection $^{129}$.

\section{CHAPITRE II : L'ADMISSIBILITÉ DE LA PREUVE PAR EXPERT}

Lorsque le juge a accepté de recevoir une preuve par expert, il doit par la suite décider de son admissibilité, c'est-à-dire du poids qu'elle aura dans l'adjudication du litige ${ }^{130}$. Nous analyserons donc les différents facteurs qui interviennent pour en déterminer l'admissibilité.

\section{Section 1: La force probante de l'expertise}

L'impact du témoignage de l'expert sur le litige peut être déterminant, alors

128. Ainsi, dans Protection de la jeunesse-323, précité, note 98, à l'étape d'établir s'il y a compromission de la sécurité et du développement de l'enfant, deux experts psychologues invités par le directeur de la protection de la jeunesse et par le père sont venus témoigner sur l'existence d'abus sexuels du père à l'égard de son enfant. Le tribunal s'est alors penché sur l'apport en preuve des témoignages contradictoires et des rapports écrits des psychologues entendus. De plus, les auteurs B. GRATTON et A. DRAGOMIR, loc. cit., note 69, 331, soulignent que : «La Loi sur la protection de la jeunesse ne limite aucunement le nombre d'experts que peut présenter une partie. Chaque partie peut donc, à sa discrétion, présenter ou non une preuve d'expert dans le cadre de l'enquête sur compromission».

129. Voir, entre autres, Protection de la jeunesse-432, précité, note 34 , où les parties s'étaient soumises à une évaluation psychologique demandée par le directeur de la protection de la jeunesse. La mère a procédé à une contre-expertise mais le père refusa de s'y soumettre. La compromission n'ayant pas été déclarée, le tribunal ne pouvait donc pas recourir aux articles 86 et 88 de la Loi sur la protection de la jeunesse pour ordonner au père de s'y soumettre. Le tribunal fut toutefois d'avis qu'en vertu de la règle audi alteram partem et qu'étant donné que le père s'était déjà soumis à l'expertise du directeur de la protection de la jeunesse, il devait conséquemment se soumettre à la contre-expertise demandée par la mère. Chaque partie doit avoir la possibilité de répondre aux prétentions de l'autre, ce qui inclut la possibilité de la contredire.

130. Notons que le nouvel article 2845 C.c.Q. précise que «la force probante du témoignage est laissée à l'appréciation du tribunal». 
que dans d'autres circonstances, le juge peut simplement le mettre de côté. Après avoir défini ce qu'on entend par force probante, nous allons étudier brièvement les facteurs les plus souvent retenus par les tribunaux pour déterminer la force probante d'une expertise.

\section{A) La notion de force probante}

La force probante est définie comme étant «la valeur ou la force de persuasion, voire de conviction que le tribunal accorde aux divers moyens de preuve qui lui ont été présentés au moment où il est appelé à évaluer la preuve» ${ }^{131}$. Celle-ci sera appréciée suivant les mêmes critères, qu'il s'agisse d'un témoin ordinaire ou d'un témoin expert ${ }^{132}$. Ainsi, la règle voulant que les témoignages se pèsent et ne se comptent pas s'applique également aux témoins experts. Il en résulte que ce n'est pas l'opinion soutenue par plusieurs experts qui sera nécessairement la plus probante, mais plutôt celle qui est la plus compatible avec l'ensemble de la preuve ${ }^{133}$.

\section{B) Les facteurs à considérer pour déterminer la force probante}

Examinons succinctement les facteurs sur lesquels le juge s'appuie pour déterminer la valeur probante d'une expertise.

\section{La crédibilité ${ }^{134}$}

Le juge pourra utiliser, pour déterminer la crédibilité du témoignage d'un expert, certains indices, telles l'intégrité générale et l'intelligence du témoin, ses facultés d'observation et la capacité de sa mémoire ${ }^{135}$, c'est-à-dire sa capacité de

131. B. GRATTON et A. DRAGOMIR, loc. cit., note $69,341$.

132. Shawinigan Engineering Company c. Naud, [1929] R.C.S. 341, $343:$ «[...] la loi ne fait aucune distinction entre les professionnels et les autres témoins. Leurs témoignages doivent être appréciés comme les autres, et le tribunal est tenu de les examiner et de les peser comme toute autre preuve faite dans la cause.»

133. H. KÉLADA, op. cit., note 8, p. 275.

134. On définit la crédibilité ainsi : «Caractère de ce qui peut être cru, de quelqu'un qui est digne de confiance» : Le Petit Larousse illustré, Paris, Larousse, 1992. Voir L. DUCHARME, Précis de la preuve, $4^{\mathrm{e}}$ éd., Montréal, Wilson et Lafleur ltée, 1993, $\mathrm{n}^{\circ}$ 508, p. 168, qui énumère certains facteurs qui peuvent influencer sur la crédibilité et non pas sa recevabilité.

135. Ces propos sont cités dans : Protection de la jeunesse-329, précité, note 77, 1746. 
rapporter fidèlement les faits.

La notion de crédibilité est intimement liée à celle d'impartialité. Le rôle de l'expert étant d'éclairer le tribunal, il doit faire preuve d'objectivité sans quoi le tribunal n'accordera que peu de poids à son opinion ${ }^{136}$. Dans cette optique, le tribunal est justifié de rejeter le rapport d'un expert lorsqu'il est manifeste qu'il a pris position en faveur de la partie qui a requis ses services ${ }^{137}$.

\section{La qualification}

Un témoin sera qualifié d'expert lorsqu'il aura démontré qu'il a acquis une compétence pertinente dans le domaine nécessitant une expertise, peu importe le moyen par lequel il a acquis ses connaissances ${ }^{138}$. C'est la valeur probante du témoignage qui en sera affectée et non sa recevabilité ${ }^{139}$ :

«L'opinion d'une personne compétente, expérimentée et impartiale est manifestement supérieure à celle du témoin qui possède peu de connaissances ou d'expérience ou qui a intérêt à favoriser un plaideur» ${ }^{140}$.

\section{Les méthodes utilisées}

La validité des tests administrés est souvent mise en doute, notamment dans les cas d'abus sexuels, parce que certaines méthodes utilisées laissent une trop grande place à l'appréciation subjective alors que d'autres ne sont pas suffisam-

136. J.-C. ROYER, op. cit., note 8, n 462, p. 170; Paterson and Sons Ltd c. Mannix Ltd, [1965] R.C.S. 180, 183, où le tribunal affirme que l'impartialité affecte seulement le poids du témoignage.

137. Droit de la famille-1288, [1989] R.D.F. 635, 640 (C.Q.).

138. H. KÉLADA, op. cit., note 8, p. 269; ainsi, un psychologue qui travaille généralement avec des personnes souffrant de problèmes d'alcool ou de drogue ne sera probablement pas qualifié dans un cas d'abus sexuel. Voir aussi MIUF-11, [1988 R.D.J. 452 (C.S.) : «[...] même si le témoin n'a pas une formation académique d'épidémiologiste, son expérience professionnelle est suffisante pour qu'il soit admis à témoigner comme expert».

139. Protection de la jeunesse-233, précité, note 89,2701, où le peu d'expérience de l'expert dans l'application des techniques des poupées sexuées est souligné et fait perdre la valeur de son expertise; voir également Mathieu c. Beauceville (Corp. de la Ville de), précité, note $99:$ «...] il n'existe aucune règle qui permette au tribunal de refuser d'entendre un témoin expert parce que sa compétence d'expert n'a pas été établie [...]. C'est la valeur probante de son témoignage et non sa recevabilité qui est en cause».

140. J.-C. ROYER, op. cit., note 8, n ${ }^{\circ}$ 478, p. 175. 
ment concluantes ${ }^{141}$. Ainsi, les tribunaux sont réticents à accorder une certaine force aux expertises tendant à démontrer qu'un présumé agresseur ou une présumée victime correspondent à un profil type ${ }^{142}$. D'une part, aucune étude sérieuse n'a pu démontrer l'existence de traits suffisamment caractéristiques pour pouvoir s'y fier ${ }^{143}$ et, d'autre part, il s'agit d'un profil abstrait alors que le juge doit décider en fonction d'un cas précis ${ }^{144}$.

Un autre test fréquemment employé en matière d'abus sexuels est l'utilisation des poupées sexuées dans le processus d'évaluation. Depuis quelques années, la force probante d'un tel test a été remise en question. Effectivement, plusieurs études soutiennent qu'il ne constitue pas une preuve valable d'abus sexuels ${ }^{145}$ puisqu'il ne semble pas y avoir de comportement typique chez les enfants victimes d'abus sexuels qui pourrait les différencier de ceux qui n'ont jamais été victimes de tels abus ${ }^{146}$.

Notons, enfin, que quel que soit le test utilisé, il en existe plusieurs autres qui peuvent être appliqués à différentes situations ${ }^{147}$ et dont l'impact sur la force probante dépend du lien de connexité entre cette méthode et le fait en litige ${ }^{148}$. Toutefois, la compétence de celui qui l'administre et sa façon de procéder peuvent faire varier grandement l'importance d'une telle preuve.

141. Ibid.

142. Pour une étude plus détaillée sur ce sujet, voir L. MORIN et C. BOISCLAIR, loc. cit., note 87, 50-52.

143. Ibid.

144. Protection de la jeunesse-539, précité, note 98, 1147, où le tribunal souscrit aux propos suivants : «Le tribunal n'a pas à statuer si ce père entre dans le cadre, la catégorie ou le profil d'un abuseur, mais bien de décider si le père en particulier et dans les circonstances qui sont soumises, a abusé de son enfant».

145. À ce sujet, voir : L. MORIN et C. BOISCLAIR, loc. cit., note 87, 54-58.

146. Ibid. Voir aussi : Michel HUARD et Claire JODOIN-PILON, «L'expertise psychologique en situation d'abus sexuel», dans L'enfant abusé : psychologie et droit (1992), Cowansville, Éditions Yvon Blais inc., 1992, p. 1, à la page 11.

147. Tels les tests d'évaluation intellectuelle et d'évaluation de la personnalité. Pour une description de ces tests, voir : M. HUARD et C. JODOIN-PILON, ibid.

148. Protection de la jeunesse-539, précité, note 98, 1146-1147. 


\section{Les qualités de l'expertise}

La façon dont s'est déroulée l'expertise peut avoir un impact déterminant sur son admissibilité. Les deux principales qualités que l'on retrouve dans une bonne expertise sont la rencontre de toutes les personnes impliquées dans le litige ${ }^{149}$ et l'objectivité de l'expert lors de la passation des tests ${ }^{150}$. En effet, le rapport d'un expert qui n'a pas rencontré toutes les personnes directement concernées par le litige sera incomplet et, par le fait même, moins probant.

Lorsque l'expertise comporte une appréciation subjective des résultats, l'expert doit s'assurer qu'il adopte une position neutre, sans quoi le tribunal pourra rejeter son rapport.

\section{L'opinion du témoin expert}

Contrairement au témoin ordinaire qui témoigne des faits dont il a eu personnellement connaissance, le témoin expert peut émettre une opinion en se basant sur des faits qui lui ont été racontés. Si ces faits sont contestés, son opinion se limitera alors aux différentes hypothèses possibles en l'espèce, à

149. L. MORIN et C. BOISCLAIR, loc. cit., note 87, 68, écrivent à ce sujet : «La condition essentielle pour faire une évaluation complète de la situation est de pouvoir rencontrer toutes les parties, incluant l'enfant, et non seulement une ou deux personnes». Ils mentionnent également qu'en certaines circonstances, par exemple en matière de garde, il peut être nécessaire d'évaluer l'enfant dans son milieu afin que l'expert puisse être en mesure de témoigner personnellement et directement de ses observations, des faits ou autres preuves cliniques devant le tribunal.

150. Lors de l'étude des limites de l'expertise inhérentes à la science, nous avons signalé que l'enfant était très vulnérable à la suggestibilité et que, malheureusement, le déroulement de l'expertise comporte souvent du matériel suggestif. L'arrêt Protection de la jeunesse-233, précité, note 89, pp. 2709 et 2710, illustre bien ce danger. Dans cette affaire, l'expert avait utilisé des poupées sexuées lors de l'entrevue avec l'enfant et filmé celle-ci afin qu'elle puisse être visionnée par le tribunal. Ce dernier s'exprime ainsi quant à la valeur probante de ce vidéo : «On assiste là à un jeu fort dirigé avec des questions qui ne suggèrent non seulement les réponses, mais qui parfois les donnent. [...] le fait qu'il fut obligé de questionner de façon si suggestive et insistante pour obtenir les réponses [...] fait perdre aux réponses des enfants leur caractère spontané et, partant, ce qui fut dit par les enfants n'était plus des déclarations, ayant perdu leur caractère volontaire. [...] la façon dont les enfants ont été amenées à répondre fait perdre toute valeur probante au vidéo et au témoignage des enfants s'y retrouvant». 
partir de questions hypothétiques ${ }^{151}$. Bref, la valeur probante de l'opinion donnée par l'expert varie selon que les faits sur lesquels elle est fondée sont prouvés ou non ${ }^{152}$.

\section{Le témoignage sur les déclarations extrajudiciaires de l'enfant}

En raison de son statut particulier, l'expert n'est pas soumis à l'interdiction du ouï-dire. Cette exception se manifeste différemment selon que l'on se trouve devant un tribunal de droit commun ou devant la Chambre de la jeunesse.

\section{a) Devant le tribunal de droit commun}

L'article 2870 C.c.Q. énonce une exception à la prohibition du ouï-dire ${ }^{153}$. En effet, les déclarations extrajudiciaires d'un enfant inapte à témoigner sont admissibles en preuve si elles satisfont aux conditions de nécessité et de fiabilité ${ }^{154}$. Il faut donc qu'il soit impossible ou déraisonnable de faire témoigner l'enfant, et les circonstances entourant ses déclarations devront donner des garanties suffisamment sérieuses pour pouvoir s'y fier. Lorsque ces deux conditions sont remplies, le témoin pourra relater en cour les propos de l'enfant et son témoignage fera preuve du contenu de ceux-ci. Par contre, le témoignage de l'enfant capable de rapporter les faits et qui comprend le devoir de dire la vérité devra, en vertu du nouvel article 2844 C.c.Q., être corroboré afin de faire preuve de son contenu.

\section{b) Devant la Chambre de la jeunesse}

En matière de protection de la jeunesse, l'exception à la prohibition du ouï-

151. D.A. BELLEMARE, loc. cit., note 63, 478.

152. La Cour suprême dans $R$. c. Abbey, précité, note 100, 46, s'est exprimée ainsi concernant la nécessité de prouver les faits sur lesquels un expert s'est basé pour faire ses recommandations : «[...] c'est à tort que le juge a tenu pour prouvés les faits sur lesquels les médecins s'étaient fondés pour former leurs opinions [...]. Pour que l'opinion d'un expert puisse avoir une valeur probante, il faut d'abord conclure à l'existence des faits sur lesquels se fonde l'opinion».

153. La règle de la prohibition du ouï-dire est contenue à l'article $2843 \mathrm{al}$ 2. C.c.Q.

154. L. DUCHARME, op. cit., note 134, $\mathrm{n}^{\circ} 1314$ à 1319, pp. 426-427; voir aussi : $R$. c. Khan, [1990] 2 R.C.S. 531. Il est à noter que l'article 2870 alinéa 3 C.c.Q. édicte que les déclarations «spontanées et contemporaines de la survenance des faits» sont présumées présenter ces garanties. 
dire est prévue par l'article 85.5 L.P.J. qui stipule que la déclaration de l'enfant antérieure à l'instance est recevable pour faire preuve de l'existence des faits qui y sont allégués lorsqu'il est inapte à témoigner ou en est dispensé par le tribunal.

Toutefois, le témoignage de l'expert sur les déclarations extrajudiciaires de l'enfant devra, contrairement à l'exception prévue à l'article 2870 C.c.Q., être corroboré par d'autres éléments qui en confirmeront la fiabilité ${ }^{155}$ afin de faire preuve de leur contenu. Notons, toutefois, que lorsque l'enfant témoigne à l'instance, l'article 85.5 L.P.J. ne s'applique pas et son témoignage n'a pas à être corroboré $^{156}$. Bref, sous la Loi sur la protection de la jeunesse, seul le ouï-dire doit être corroboré ${ }^{157}$.

\section{La confection du rapport : distinction entre les faits et l'opinion de l'expert}

La qualité du rapport de l'expert est un facteur important susceptible d'influencer sa force probante. En effet, la lecture de la jurisprudence nous apprend que les juges sont plus réceptifs à une opinion d'expert lorsque celle-ci est bien articulée ${ }^{158}$.

De plus, le rapport de l'expert qui distingue bien les faits, son interprétation et ses recommandations à partir des faits qu'il a constatés, sera généralement mieux accueilli par le tribunal. Un tel rapport permet au juge de vérifier si les conclusions de l'expert sont fondées sur des faits prouvés et il peut ainsi en mesurer aisément la force probante.

155. $\quad$ Protection de la jeunesse-593, J.E. 93-297 (C.Q.), pp. 30-32.

156. À cet effet, voir, entre autres : Protection de la jeunesse-434, précité, note 59, 1193; Protection de la jeunesse-501, [1991] R.J.Q. 1800, 1803 (C.Q.); Protection de la jeunesse659, précité, note 86, 21; Protection de la jeunesse-649, J.E. 94-11 (C.Q.), p. 21.

157. L. MORIN et C. BOISCLAIR, loc. cit., note 87, 43-49.

158. Droit de la famille-1381, précité, note 6, 133, donne un exemple d'une expertise «bien articulée». À ce propos, les auteurs L. Gélinas et B.M. Knoppers, loc. cit., note 2, 74, énumèrent cinq éléments que devrait toujours contenir un rapport d'expert en matière familiale : «1) une évaluation psychologique et intellectuelle des parents et de l'enfant; 2) des entrevues avec toutes les personnes impliquées dans la cause; 3 ) une observation directe des interactions familiales; 4) si nécessaire, des rencontres avec les personnes appelées à côtoyer l'enfant : grands-parents, professeurs, etc.; 5) et finalement les conclusions cliniques tirées de l'ensemble de ces informations». 


\section{Section 2 : $\quad$ La contradiction entre experts}

Le tribunal est souvent confronté à des témoignages et des rapports d'experts qui sont contradictoires ${ }^{159}$, tant au plan des opinions qu'à celui des recommandations. Nous verrons tout d'abord les différentes raisons pouvant expliquer pourquoi des personnes compétentes et spécialisées ne sont pas du même avis, et nous ferons état des différents moyens dont dispose le juge pour pallier cette situation.

\section{A) Les explications possibles des expertises contradictoires}

Une des explications possibles aux expertises contradictoires résulte du défaut des experts de rencontrer toutes les parties concernées par le litige. L'expert qui ne rencontre les parties qu'individuellement ou ne les rencontre qu'ensemble peut, en certains cas, fournir au juge une opinion incomplète puisqu'elle ne tient pas compte de tous les facteurs pertinents. À cet effet, le professeur C. Boisclair note qu'en réalité, la «contradiction ne sera qu'apparente puisque les expertises n'auront pas évalué les mêmes facteurs» ${ }^{160}$.

Les théories et conceptions professionnelles peuvent également amener des expertises contradictoires dont les conclusions peuvent être souvent influencées ${ }^{161}$ par l'éducation, le milieu de vie ou de travail qui finissent par affecter les conceptions partagées ou soutenues par les experts.

\section{B) Les moyens à la disposition du juge en cas d'expertises contradictoires}

Lorsque l'écart entre les rapports est considérable, il est possible pour le juge

159. Voir à titre d'exemples: Protection de la jeunesse-323, précité, note 98; Droit de la famille1288, précité, note 137; Protection de la jeunesse-548, J.E. 92-801 (C.Q.); Protection de la jeunesse-578, J.E. 92-1710 (C.Q.).

160. C. BOISCLAIR, op. cit., note 49, p. 159.

161. L. MORIN et C. BOISCLAIR, loc. cit., note 87, 64 : «Celles-ci peuvent, dans certains cas, expliquer les contradictions, sans compter que l'interprétation des déclarations, des tests, des faits cliniques pourra conduire un expert à accepter, par exemple, que certains gestes à connotation sexuelle ne soient pas considérés comme abus sexuel, alors que pour un autre, les mêmes gestes en constituent un». Voir à titre d'exemples : Protection de la jeunesse-380, précité, note 83; Protection de la jeunesse-584, [1993] R.J.Q. 274 (C.Q.), où le tribunal est confronté à des expertises contradictoires quant à la commission d'abus sexuels. 
de requérir l'avis supplémentaire d'un autre expert pour départager ces opinions ${ }^{162}$. Le rapport de cette contre-expertise ordonnée par le tribunal sera possiblement plus complet ou, à tout le moins, plus impartial puisqu'il sera demandé par le tribunal.

La seconde alternative qui s'offre au juge en cas d'expertises contradictoires consiste à s'en remettre à la preuve profane. L'analyse de la jurisprudence à ce sujet montre que les tribunaux n'hésitent pas à s'appuyer sur la preuve profane lorsque celle présentée par les experts est contradictoire ${ }^{163}$.

\section{Section 3 : Le degré de preuve requis pour donner effet à l'expertise}

En matière civile, c'est la règle de la prépondérance des probabilités qui prévaut $^{164}$. Toutefois, le degré de probabilité a longtemps été l'objet de discussion en raison de la confusion entourant la variable du degré de probabilité. Cette dernière concerne l'appréciation de la qualité de la preuve à l'intérieur de l'opération de détermination de la prépondérance des probabilités $^{165}$.

Le degré de prépondérance des probabilités est inflexible, c'est-à-dire que le juge doit être convaincu à $51 \%$ des prétentions d'une partie, alors que le degré de probabilité requis, pour donner effet à l'expertise, varie selon la gravité de

162. C.p.c., articles 414 et suivants.

163. À cet effet, voir notamment Michaud c. Bergeron, [1980] C.A. 246, où le juge trouve normal de faire entrer en ligne de compte le témoignage des témoins ordinaires puisqu'il n'avait aucune raison de faire prévaloir l'opinion des experts du défendeur sur celle des experts du demandeur. De même, dans Protection de la jeunesse-207, J.E. 86-384 (T.J.), le tribunal ne voyait pas la nécessité de départager les opinions contradictoires des experts ni la nécessité de nommer un autre expert conformément aux articles 414 et suivants du C.p.c. puisque la preuve apportée par les profanes permettait de conclure aux abus sexuels. Voir également Droit de la famille-1661, 1992] R.J.Q. 2479 (C.S.).

164. C.c.Q., art. 2804. Voir à ce sujet : Parent c. Lapointe, [1952] 1 R.C.S. 376, 380; Mutuelle du Canada c. Aubin, [1979] 2 R.C.S. 298; Mercantile (Compagnie d'assurance) c. Pigeon, [1966] R.L. 527 (C.S.); Mignault c. Habel, [1971] R.P. 337 (C.S.); Sous-ministre du Revenu de la province de Québec c. Jobin, [1971] C.S. 565; Protection de la jeunesse-380, [1990] R.J.Q. 529 (C.S.).

165. Jean-Guy BERGERON, Les contrats d'assurances, t. 2, Sherbrooke, Les éditions SEM inc., 1992, p. 48; American Home Ass. Co. c. Auberge des Pins inc., [1990] R.R.A. 152 (C.A.). 
chaque situation ${ }^{166}$, sans pour autant mettre en doute le critère de la prépondérance de la preuve ${ }^{167}$. Ainsi, il n'y a pas de critère intermédiaire, comme la forte balance des probabilités, qui se situerait entre celui du droit criminel (hors de tout doute raisonnable) et celui du droit civil (la prépondérance de la preuve). La prépondérance est donc elle-même une conclusion ne souffrant pas de variable : elle existe tout simplement ou elle n'existe pas.

\section{Section 4: L'utilisation de l'expertise par les tribunaux}

Les tribunaux se sont parfois montrés réticents à l'égard de l'expertise puisqu'ils craignaient que les experts décident du litige à leur place, leur faisant ainsi perdre le contrôle sur l'issue du litige ${ }^{168}$. Ceux-ci acceptent aujourd'hui le principe de l'expertise, considérant qu'ils ont entière discrétion à l'égard de celleci. En effet, les tribunaux ont toujours la faculté de refuser ou d'accepter une expertise, n'étant en aucun cas liés par celle-ci ${ }^{169}$, puisqu'il revient au juge seul d'apprécier la preuve ${ }^{170}$. Une fois précisés les rôles de chacun au fil des années, l'expertise est reçue aujourd'hui comme un moyen pouvant aider le juge et non comme un substitut à son appréciation de la preuve.

166. La preuve faite selon la balance des probabilités peut donc comporter différents degrés de probabilités. Donc, si nous sommes en présence d'une allégation d'abus sexuels, le tribunal exigera une plus grande probabilité pour tenir comme prouvé l'abus, tout en jugeant du litige final selon la prépondérance de la preuve. La Cour suprême a d'ailleurs reconnu, dans The Continental Insurance Co. c. Dalton Cartage Co. Ltd, [1982] 1 R.C.S. 164, 170, que la norme de la prépondérance des probabilités pouvait comporter différents degrés.

167. C'est d'ailleurs ce que soutient la Cour suprême dans R. c. Oakes, [1986] 1 R.C.S. 103, 137, de même que dans les arrêts suivants : Bater c. Bater, [1950] 2 All E.R. 458, 459; Protection de la jeunesse-558, J.E. 92-1123 (C.S.); Protection de la jeunesse-548, précité, note 159, 40; L. DUCHARME, op. cit., note 134, n ${ }^{\circ}$ 148, p. 55.

168. Marie-Josée BLONDIN, Diane DÉSY, Francine DESROCHERS, Annette LE MONNIER, Carmen LUSSIER, «Évolution jurisprudentielle (1950-1983) du critère de la conduite des conjoints dans l'attribution de la garde des enfants», (1986) $46 R . d u$ B. 105, 126 : «On constate, par ailleurs, que dans certains cas, les juges sont peu enclins à se servir de ces expertises. L'attribution de la garde étant de leur compétence, peut-être craignent-ils de se départir d'une partie de leurs pouvoirs en suivant les recommandations des experts».

169. Shawinigan Engineering Co. c. Naud, précité, note 132; Donolo inc. c. St-Michel Realties Inc., précité, note 7; X. c. Z., précité, note 3; Droit de la famille-527, [1988] R.J.Q. 1945 (C.S.); Droit de la famille-1632, 1992 R.D.F. 524 (C.S.); H. KÉLADA, op. cit., note 8, p. 99; Jean-Paul LANDRY, «De la preuve par expert : la jurisprudence», (1980) $40 R . d u B$. $652,656$.

170. Alain BERNARDOT, et Robert KOURI, La responsabilité civile médicale, $3^{\mathrm{e}}$ éd., Cowansville, Éditions Yvon Blais inc., 1990, nº 27, p. 17. 


\section{TROISIÈME PARTIE : POUR UNE MEILLEURE UTILISATION DE L'EXPERTISE : CRITIQUES CONSTRUCTIVES EN REGARD DU DROIT ÉTRANGER}

Nos recherches en droit comparé nous ont confirmé l'intérêt du recours à l'expertise en raison des grandes similitudes entre le Québec, le reste du Canada et les États-Unis. En effet, la mise en oeuvre du droit à l'expertise est sensiblement la même dans les deux pays. Il n'en demeure pas moins que nous avons rencontré principalement trois difficultés auxquelles il serait possible d'apporter des correctifs. À cet effet, nous avons identifié certaines différences intéressantes provenant de certains États américains, plus particulièrement celui de la Californie, qui pourraient être incorporées au droit québécois afin de faire progresser et d'améliorer le rendement et l'efficacité de la preuve par un expert du Québec.

Aux États-Unis, cinq États sont considérés comme les précurseurs lorsqu'il s'agit d'intervention sociale ${ }^{171}$, alors que les autres ont plutôt tendance à suivre leur politique. L'État californien se retrouve au premier plan en raison de ses politiques d'avant-garde ${ }^{172}$. En effet, la Californie a démontré dans le passé la volonté de moderniser ses règles de preuve afin d'éviter l'injustice découlant de l'application trop rigide de celles-ci ${ }^{173}$. Les tribunaux ont de plus senti le besoin de ne pas s'astreindre à appliquer des règles contraires à la logique et d'opter pour le pragmatisme et le bon sens dans l'application des règles de preuve ${ }^{174}$.

171. Ces États sont respectivement la Californie, la Floride, Washington, le Colorado et le Connecticut.

172. Pour des raisons démographiques cependant, la Floride devient de plus en plus importante et pourrait bientôt surpasser la Californie.

173. People v. Collup, 27 Cal. App. 2d 829 (1946).

174. California Bank v. Diamond, 301 P. 2 d 60 (1956); Los Angeles v. Faus, 312 P. 2d 680 (1957). 


\section{CHAPITRE I : L'IMPACT NÉGATIF D'UN TROP GRAND NOMBRE D'EXPERTISES}

L'expertise en matière familiale se situe habituellement dans une période de crise qui provoque généralement de l'anxiété chez la personne concernée, particulièrement lorsqu'il s'agit d'un enfant, d'où l'importance de procéder à l'expertise avec diligence et professionnalisme afin d'être efficace et d'en limiter l'impact négatif.

\section{Section 1: Précisions sur la problématique de la multiplication des expertises}

Le nombre élevé d'experts impliqués dans un dossier ${ }^{175}$ paraît parfois inquiétant puisqu'une telle pratique se fait souvent au détriment de la personne qui est soumise à plusieurs évaluations en présence de divers intervenants ${ }^{176}$. De plus, le nombre élevé d'experts ne sert pas les fins de la justice puisqu'il entraîne inévitablement des expertises contradictoires et, par le fait même, l'expert ne joue plus son rôle de guide auprès du tribunal.

Il ne serait toutefois pas approprié d'exclure l'expertise du débat contradictoire et de n'accorder qu'au tribunal seul le pouvoir de requérir une expertise $^{177}$. Cela porterait atteinte au droit fondamental des parties de faire valoir leurs prétentions de façon pleine et entière. De plus, même si la présence d'un seul expert faisait disparaître le problème des expertises contradictoires,

175. Dans l'affaire Protection de la jeunesse-380, précité, note 83, et Protection de la jeunesse380 , précité, note 164, pas moins de neuf experts furent entendus par la cour. Dans Droit de la famille - 1242, précité, note 2, alors que depuis 1985 dix-sept juges avaient été amenés à se pencher sur l'odyssée des parties dans ce dossier, l'enfant en était rendu à menacer son père : «Je vais le dire à Vermette (le psychologue)».

176. Les propos du tribunal dans Protection de la jeunesse-380, précité, note 83, 1043, sont révélateurs des effets néfastes d'une expertise gynécologique : «Si la justice a à s'améliorer au niveau de l'humanisation de ses rapports avec les gens, la médecine a à s'améliorer pour beaucoup dans son humanisation. Je veux dire : une enfant de cinq (5) ans qui se fait photographier avec diapositives, etc.... et des appareils si près, je veux dire : je n'ai pas de doute qu'il peut rester des marques chez un enfant.»

177. Comme le suggère D.A. BELLEMARE, loc. cit., note 63, 483-485; voir D. JUTRAS, op. cit., note 93, pp. 900-902, où l'auteur se prononce sur le problème des expertises contradictoires et sur les différentes solutions proposées. Pour sa part, l'auteur soutient que peu importe la solution envisagée, le problème des expertises contradictoires est inévitable. 
cela pourrait toutefois priver le tribunal d'informations essentielles ${ }^{178}$.

\section{Section 2: Le pouvoir du juge de limiter le nombre d'expertises}

Le droit californien, qui accorde au juge le pouvoir de limiter le nombre d'experts, nous apparaît être la solution appropriée aux effets négatifs résultant d'un trop grand nombre d'expertise ${ }^{179}$. La principale difficulté consiste à identifier les situations propices à une intervention du tribunal puisqu'il peut être difficile de décider quels témoignages sont superflus ou inutiles. Pour cette raison, le tribunal n'exclura généralement que les témoignages additionnels sur la même question ou sur le même fait controversé. Mentionnons que cette règle doit évidemment être appliquée aux deux parties afin qu'aucune ne soit injustement avantagée par son application.

\section{Section 3 : Le rapport de l'expert tenant lieu de preuve en lieu et place de son témoignage}

Il serait également possible de contrer les désavantages du grand nombre d'experts en s'inspirant d'une règle de droit de la Colombie-Britannique. Le Evidence Act of British Columbia suggère une alternative à la preuve par experts. Dans un premier temps, une partie peut déposer le rapport de l'expert afin qu'il fasse preuve de son contenu ${ }^{180}$ et toute partie pourra appeler l'expert à venir témoigner en cour. Le droit au contre-interrogatoire est ainsi préservé. Dans un deuxième temps, une partie peut décider de faire témoigner l'expert et ce sera alors son témoignage qui fera preuve de son contenu en dépit du fait que son rapport, déposé préalablement, soit différent ${ }^{181}$. Il est important de souligner que la première possibilité est particulièrement intéressante puisqu'elle permet

178. Protection de la jeunesse-649, précité, note 156,11, où le tribunal estime qu'il a été avantageux d'avoir l'opinion de plus d'un expert.

179. Cal. Evid. Code $\S 723$ (1966). La solution californienne nous semble appropriée en ce qu'elle permettrait de limiter le nombre de témoins experts lorsque le tribunal serait satisfait de la preuve qui lui a été présentée. Ce faisant, cela permettrait au moins de freiner les abus qui ont pour effet d'augmenter la durée du procès et la complexité du litige. Mentionnons toutefois qu'en vertu de la Loi sur le divorce, L.R.C. (1985), c. 3 ( $2^{\mathrm{e}}$ suppl.), c'est la Loi sur la preuve au Canada, L.R.C. (1985), c. C-5, qui s'applique et que le nombre de témoins est limité à 5 en ce cas.

180. Evidence Act, R.S.B.C. 1979, c. 116, art. 10 (ci-après citée «E.A.»).

181. E.A., art. 11. 
d'éviter des pertes de temps parfois considérables, tout en sauvegardant le droit à la contre-expertise.

\section{CHAPITRE II : L'INCERTITUDE RÉSULTANT DE L'ABSENCE DE DIRECTIVES IDENTIQUES PRÉALABLES DONNÉES AUX EXPERTS}

L'absence de directives adéquates données aux experts constituent l'une des principales causes des opinions divergentes ${ }^{182}$. En effet, les experts, ayant reçu des mandats différents, arriveront probablement à des conclusions différentes. Étant donné la diversité des questions auxquelles les experts peuvent être appelés à répondre, comme, à titre d'exemples : s'agit-il de droit d'accès ou de droit de garde? Faut-il déterminer si le parent a le profil d'abuseur ou s'il a effectivement abusé sexuellement son enfant ${ }^{183}$ ? Il est conséquemment très important de circonscrire le mandat qu'on leur confie et de s'assurer qu'ils ont tous reçus les mêmes directives et qu'ils doivent tous se prononcer sur la même question. À cette fin, il serait opportun d'inclure un volet concernant la psychologie dans le cours de la formation des futurs représentants de l'enfant devant les tribunaux tel que recommandé récemment par le Barreau du Québec ${ }^{184}$. Ces derniers seraient alors plus en mesure de donner un mandat clair aux experts et de distinguer une expertise pertinente au litige de celle qui ne ferait qu'encombrer le débat ${ }^{185}$. Même si les experts reçoivent les mêmes directives, cela n'éliminera toutefois pas tous leurs désaccords, puisqu'ils pourront alors résulter d'une interprétation différente des directives ou de l'approche qu'ils auront adoptée ${ }^{186}$, mais tous iront au moins dans la même direction afin de mieux servir les intérêts des parties et de l'administration de la justice.

182. C. BOISCLAIR, op. cit., note 49, p. 159.

183. Paule LAMONTAGNE, «L'expertise psycho-légale au tribunal de la famille (Cour supérieure)» dans Les enfants devant la justice - juges, avocats et experts témoignent, Cowansville, Éditions Yvon Blais inc., 1990, p. 257, à la page 262.

184. La représentation des enfants par avocat, Barreau du Québec, 1994, pp. 55 à 58, où l'on traite de la formation universitaire et professionnelle.

185. L. MORIN, loc. cit., note 79, 604.

186. Voir à ce sujet le Chapitre III de la Partie I. 


\section{CHAPITRE III : LES TESTS SANGUINS DANS LES ACTIONS EN RECHERCHE DE PATERNITÉ}

La dernière amélioration que nous proposons a trait à l'expertise sanguine. À cet égard, le California Uniform Act on Blood Tests to Determine Paternity ${ }^{187}$ permet au tribunal d'ordonner à une personne de se soumettre à un test sanguin afin de déterminer la filiation d'un enfant ${ }^{188}$. Les résultats de ce test ne seront toutefois admis qu'à une fin d'exclusion de paternité ${ }^{189}$. Signalons, à ce propos, que d'autres États américains adoptent la même position (notamment le Connecticut et l'Ohio), tandis que d'autres en permettent l'utilisation autant aux fins d'exclure que d'établir la paternité (mentionnons le Colorado, l'État d'Hawaï, l'Indiana, le Dakota du Nord). Dans le Kentucky, la Louisiane et le Maine, le juge a discrétion pour admettre une telle preuve, sous réserve de certaines conditions.

Lorsque les tests concluent à la paternité, les tribunaux de la Californie ne les admettront pas en preuve, ils chercheront plutôt des indices dans le reste de la preuve ${ }^{190}$. Nous croyons l'approche des États admettant en preuve l'expertise sanguine, qu'elle soit négative ou positive, plus réaliste et conforme au développement technologique qui rend ces tests certains à 99.8\% lorsqu'il s'agit

187. Cal. Evid. Code $§ 895$ (1968).

188. Contrairement à d'autres moyens de preuve qui sont susceptibles de faire l'objet d'interprétation, et conséquemment de résultats contradictoires, les résultats de tests sanguins sont difficiles à réfuter s'ils sont obtenus dans des conditions propices par des experts qualifiés.

189. En effet, le California Act a omis la disposition de l'Uniform Act (loi fédérale) qui prévoyait que la preuve des tests sanguins pouvait être admise en preuve pour établir la paternité. Cette disposition était la suivante : «If the court finds that the conclusions of all the experts, as disclosed by the evidence based on the [blood] tests, are that the alleged father is not the father of the child, the question of paternity shall be resolved accordingly. If the experts disagree in their findings or conclusions, the question shall be submitted upon all the evidence». Les tribunaux ont décidé que la législation ne permettait l'admissibilité des tests sanguins que pour exclure la paternité, puisque la disposition permettant l'admission en preuve des tests avait été omise délibérément. Dodd v. Henkel, 148 Cal Rptr 780 (1978).

190. À cet effet, notons que dans plusieurs États, dont la Californie, que les traits physiologiques de deux personnes peuvent être mis en preuve afin de servir d'indices de paternité. Il fut cependant décidé, au Colorado, que l'exhibition d'un enfant devant le tribunal afin de faire la preuve de leur ressemblance irait à l'encontre du droit à la contre-expertise puisqu'une telle preuve serait faite sans le concours d'un expert. 
de déterminer la filiation d'une personne ${ }^{191}$. Il ne paraît donc plus justifié de distinguer la preuve positive de la preuve négative de paternité. Le législateur québécois devrait tenir compte des progrès scientifiques et permettre aux tribunaux d'ordonner une expertise sanguine dans la recherche de la paternité. Une prise de sang ne porte pas plus atteinte aux droits de la personne que l'alcotest lorsqu'il y va de l'ordre public. En effet, l'établissement de la paternité concerne l'ordre public puisqu'il libère l'État de l'obligation de pourvoir financièrement aux soins de l'enfant ${ }^{192}$. Il est grand temps que le principe de l'inviolabilité de la personne humaine cesse de servir de protection à un père présumé et cède le pas devant l'ordre public et l'intérêt de l'enfant à voir sa véritable filiation établie.

\section{CONCLUSION}

Plus personne ne songe aujourd'hui à remettre en cause l'expertise au sein $\mathrm{du}$ processus judiciaire, et plus particulièrement dans le domaine familial, notamment à cause d'un plus grand dévoilement de situations de compromission et de la mutation de la famille contemporaine. Les tribunaux n'hésitent plus à recourir à des spécialistes du comportement humain afin d'être informés de toutes les implications d'une situation pour prendre la meilleure décision.

La preuve apportée par l'expert doit toutefois être placée en perspective avec l'ensemble de la preuve. En effet, la science, et spécialement celle ayant pour objet l'étude du comportement humain, comprend certaines limites auxquelles les avocats, comme les juges, doivent être sensibilisés. Ainsi, la validité des tests utilisés, la nouveauté de la technique privilégiée, les conceptions et préjugés du professionnel ainsi que l'attitude de la personne soumise à l'expertise, sont autant de facteurs qui peuvent influencer les conclusions auxquelles arrive un expert. Certaines différences entre le droit et la science, dont le degré de certitude requis est la plus significative, peuvent également avoir une influence importante à la fois sur l'expertise et sur la prise de décision

191. «Le test d'ADN est de plus en plus utilisé pour déterminer la paternité», La Presse, Montréal, le 18 février 1994.

192. En Alberta, les mères célibataires réclamant l'aide sociale doivent subroger le gouvernement dans leurs droits de poursuivre le père afin que les services sociaux puissent lui réclamer une pension alimentaire pour et au nom de la mère. «Le test d'ADN est de plus en plus utilisé pour déterminer la paternité», La Presse, Montréal, le 18 février 1994. 
par les tribunaux. C'est pourquoi le tribunal doit être attentif à ces distinctions parce qu'il lui appartient seul d'accepter ou de rejeter une expertise.

L'aide considérable apportée par les experts a réussi à vaincre les réserves que les tribunaux entretenaient jadis à leur égard. Toutefois, l'étude de la doctrine et de la jurisprudence montre que l'intérêt toujours grandissant pour l'expertise n'est pas sans engendrer certains inconvénients qui, sans remettre en cause le principe de l'expertise, justifieraient certaines modifications. La famille est une institution trop importante pour négliger de s'intéresser à tout ce qui est susceptible d'en favoriser la protection. C'est ainsi que le droit devrait adapter au contexte familial l'application de principes fondamentaux, tels le droit à l'inviolabilité de la personne humaine et le droit à la contre-expertise, afin de tenir compte des progrès scientifiques et techniques qui pourraient justifier de s'écarter de la règle de principe lorsqu'un intérêt supérieur est en cause.

L'un des principaux obstacles à surmonter est le phénomène de la multiplication des expertises. Bien que nous considérions comme indiscutable le droit à l'expertise, il y a lieu de proposer des règles pour circonscrire la portée de ce droit et limiter les inconvénients résultant d'un trop grand nombre d'expertises, telles l'augmentation du nombre des entrevues et la prolongation indue des délais. À cette fin, le tribunal devrait être investi expressément du pouvoir de limiter le nombre d'experts lorsqu'il en résulte des interventions peu utiles, n'apportant aucun élément nouveau dans le litige.

Un encadrement plus strict des spécialistes, appelés à intervenir dans un litige en matière familiale, constituerait une autre solution intéressante aux problèmes résultant de la multiplication des expertises. Dans cette perspective, les praticiens du droit en matière familiale devraient recevoir une formation particulière, dont certaines notions de psychologie, des connaissances de base sur les techniques les plus courantes d'évaluation, comme l'évaluation microscopique et macroscopique, afin de leur permettre d'apprécier si l'expertise est complète et de la contester si tel n'est pas le cas ${ }^{193}$.

Le droit à l'expertise n'est pas le seul aspect qui devrait être réaménagé. Le

193. Ainsi, l'expert qui n'aurait rencontré qu'un seul des parents peut difficilement prétendre que seul ce dernier est apte à recevoir la garde d'un enfant puisqu'il n'a pas évalué les liens entre cet enfant et l'autre parent. Il en serait de même s'il n'avait pas rencontré l'enfant. 
droit à l'inviolabilité de la personne humaine devrait comporter d'autres exceptions pour couvrir certaines hypothèses en matière familiale puisque l'application stricte de ce principe conduit, à l'occasion, à des situations pour le moins contestables. Il est difficile de comprendre, à la lumière du droit comparé, l'obstination du législateur qui refuse de rendre obligatoire l'expertise sanguine dans les actions en recherche de filiation. Cette dérogation est justifiée par un intérêt supérieur et il est douteux qu'un prélèvement sanguin puisse constituer une atteinte grave au principe de l'inviolabilité de la personne humaine. Si les réticences du législateur persistent, les tribunaux devraient, à tout le moins, être expressément autorisés à tirer une présomption défavorable du refus injustifié de se soumettre à une telle expertise. L'ordre public et l'intérêt social à ce que l'enfant connaisse sa filiation rendraient légitime une telle modification. Malgré l'entrée en vigueur d'un nouveau Code civil au début de l'année 1994, le législateur québécois accuse un retard sur plusieurs législations étrangères qui ont compris l'apport inestimable que peut constituer une expertise sanguine positive ou négative devenue aujourd'hui quasi certaine. Cette situation nous laisse perplexe, d'autant plus que dans certains domaines on accepte de déroger à l'inviolabilité de la personne humaine, notamment lorsque des intérêts pécuniaires sont en jeu. Le législateur aurait-il deux poids, deux mesures?

En terminant, il serait intéressant d'étudier la possibilité d'étendre le dépôt du rapport prévu pour les médecins à l'article 294.1 C.p.c. aux autres experts, tels les psychologues, les criminologues, les travailleurs sociaux, etc. sans que ceux-ci n'aient à témoigner ${ }^{194}$. En effet, devant le grand nombre d'experts entendus dans certains dossiers, il y a lieu de chercher des solutions qui, tout en conservant le droit de chaque partie de faire valoir ses prétentions, permettraient de limiter les inconvénients découlant de l'exercice de ce droit. Les délais prolongés résultant de l'audition de témoins supplémentaires ne font, bien souvent, qu'augmenter la tension déjà existante au sein de la famille. Le droit comparé nous incite à proposer une telle modification législative qui contribuerait à limiter la durée des procès, et du même coup à faciliter les relations entre les membres d'une famille aux prises avec des problèmes émotifs et légaux. Toutefois, cette solution ne pourrait atteindre son objectif que dans

194. Les nouveaux articles 2869 à 2874 C.c.Q. concernent, à première vue, les déclarations faites à un expert et non les opinions de l'expert (voir, entre autres, 2873 et 2874 C.c.Q.). 
la mesure où le rapport de l'expert est facilement accessible, au plan de la terminologie, aux avocats, aux juges et aux parties, sans quoi l'expert devra venir expliquer devant le tribunal le contenu et les conclusions de son expertise.

Les conséquences d'une «erreur judiciaire» dans le domaine familial peuvent avoir des répercussions catastrophiques sur la vie d'une famille, comme le tribunal le fait remarquer dans une affaire d'abus sexuels, qu'il est possible d'étendre à tout le domaine de la famille :

«L'une des pires choses qui puisse arriver à un enfant est d'être victime d'abus sexuels. L'une des pires choses qui puisse arriver à un adulte est d'être accusé à tort d'un crime aussi grave» ${ }^{195}$.

C'est pourquoi la collaboration entre le droit et la science est indispensable dans le contexte moderne des litiges familiaux. De plus, cette collaboration doit tenir compte des différences fondamentales qui distinguent chacune des disciplines afin d'en arriver à une collaboration toujours plus étroite dans la recherche de la vérité et du bien-être commun.

195. Voir Luc MORIN, «Les profils en matière d'allégations d'abus sexuels : une invitation à la prudence», (1993) R.D.U.S. 415, 434. 